Interfaces and Free Boundaries 12 (2010), 85-119

DOI $10.4171 / \mathrm{IFB} / 228$

\title{
Mixed finite element method for electrowetting on dielectric with contact line pinning
}

\author{
SHAWN W. WALKER \\ Department of Mathematics, New York University, \\ 251 Mercer Street, New York City, N.Y. 10012-1185, USA \\ E-mail:walker@cims.nyu.edu \\ ANDREA BONITO \\ Department of Mathematics, Texas A\&M University, \\ 3368 TAMU, College Station, TX 77843-3368, USA \\ E-mail: bonito@math.tamu.edu \\ RICARDO H. NOCHETTO \\ Department of Mathematics, University of Maryland, Mathematics Building, \\ College Park, MD 20742-4015, USA \\ E-mail:rhn@math.umd.edu
}

[Received 10 February 2009 and in revised form 8 January 2010]

\begin{abstract}
We present a mixed finite element method for a model of the flow in a Hele-Shaw cell of 2-D fluid droplets surrounded by air driven by surface tension and actuated by an electric field. The application of interest regards a micro-fluidic device called ElectroWetting on Dielectric (EWOD). Our analysis first focuses on the time discrete (continuous in space) problem and is presented in a mixed variational framework, which incorporates curvature as a natural boundary condition. The model includes a viscous damping term for interface motion, as well as contact line pinning (sticking of the interface) and is captured in our formulation by a variational inequality. The semi-discrete problem uses a semiimplicit time discretization of curvature. We prove the well-posedness of the semi-discrete problem and fully discrete problem when discretized with iso-parametric finite elements. We derive a priori error estimates for the space discretization. We also prove the convergence of an Uzawa algorithm for solving the semi-discrete EWOD system with inequality constraint. We conclude with a discussion about experimental orders of convergence.
\end{abstract}

\section{Introduction}

The ability to manipulate fluids at the micro-scale is an important tool in the area of bio-medical applications. Micro-fluidic devices often exploit surface tension forces to actuate or control liquids $[32,17,26]$ by taking advantage of the large surface-to-volume ratios found at the micro-scale. This paper is concerned with developing a mixed finite element method to simulate droplet motion in a micro device driven by Electrowetting-On-Dielectric (EWOD) [15, 50, 6, 43], which consists of two closely spaced parallel plates with a droplet bridging the plates and a grid of square electrodes embedded in the bottom plate [56]. Applying voltages to the grid allows the droplet to move, split, and rejoin within the narrow space of the plates. Applications range from mass spectrometry [58, 45], to 'lab-on-a-chip' [47, 31], and particle separation/concentration control [14, 55].

We describe a mixed variational formulation of a 2-D model of EWOD driven flow (see [56, 54, 57), which is discretized by finite elements. The model is similar to Hele-Shaw flow with 
a modification of the boundary condition to account for the electrical surface tension effect. In addition, the model has a frictional effect [57] due to three-phase contact line pinning and hysteresis, and is included in our formulation as a variational inequality with viscous damping.

Other computational models of electrowetting exist: [39] assumes quasi-static behavior of the droplet; [42] uses a diffuse interface method to simulate droplet motion in a scaled up version of the EWOD device; [24] uses a phase-field method. Other methods [5, 44] use a Volume of Fluid (VoF) technique to track droplet motion but do not give precise information about the liquid-gas interface shape. Lastly, none of these methods include the lossy effect of contact line friction nor the pinning effect.

The rest of the paper is outlined as follows. Section 2 briefly discusses the governing equations for droplets driven by EWOD, as well as the nonlinear liquid-gas interface friction model. Next, we describe our time discretization in Section 3 followed by its well-posedness in Section 4 We formulate a space discretization for the EWOD problem in Section 5, discuss its well-posedness and prove a priori error estimates for the continuous in space problem. Section 6 describes the convergence of an Uzawa method for solving the nonlinear time-discrete system. Finally, we present experimental orders of convergence in Section 7

\section{Electrowetting model}

\subsection{Governing equations in the bulk}

The governing equations for the flow of viscous incompressible liquid between the parallel plates of an EWOD device is similar to Hele-Shaw [33], [4] type flow with pressure boundary conditions at the liquid-gas interface proportional to its curvature with added forcing and interfacial friction terms. The details can be found in [56], [54]. The 2-D flow equations in the bulk of the droplet $\Omega=\Omega(t)$ are given by

$$
\begin{aligned}
\alpha \partial_{t} \mathbf{u}+\beta \mathbf{u}+\nabla p=0 & \text { in } \Omega, \\
\nabla \cdot \mathbf{u}=0 & \text { in } \Omega,
\end{aligned}
$$

where $\mathbf{u}$ is the vector velocity field (in the plane of the device) and $p$ is the pressure. The time derivative $\partial_{t} \mathbf{u}$ in (1) is unusual in Hele-Shaw models and incorporates inertial effects; its magnitude may be large due to rapidly varying pressure boundary conditions if high frequency voltage actuation is used to modulate the droplet's contact angles. The nondimensional constants $\alpha$ and $\beta$, where $\alpha \ll \beta$, depend on fluid parameters and device geometry.

\subsection{Boundary conditions}

The boundary conditions for the bulk equations in (1) are

$$
p=\kappa+E \quad \text { on } \Gamma,
$$

where $\kappa$ is the curvature of the 1-D boundary $\Gamma$ of the 2-D droplet (i.e. the liquid-gas interface) and $E$ is a given function on the boundary that captures the ability of EWOD to locally modulate the boundary pressure through voltage actuation [56]. The electric forcing $E(\mathrm{x})$, for $\mathrm{x}$ in $\Gamma$, is a function of the electrode pad voltage at the point $\mathbf{x}$. 


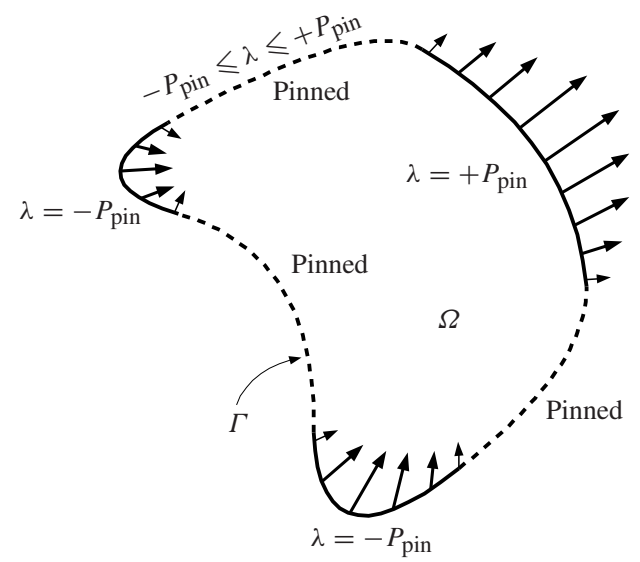

FIG. 1. A 2-D droplet $\Omega$ with parts of the boundary $\Gamma$ pinned. The pinned regions are denoted by a dashed line; unpinned regions are shown as a solid line with velocity arrows indicating direction of motion. An outward motion is considered positive $\left(\mathbf{u} \cdot \boldsymbol{\nu}>0 ; \lambda=+P_{\text {pin }}\right)$, and an inward motion is negative $\left(\mathbf{u} \cdot \boldsymbol{\nu}<0 ; \lambda=-P_{\text {pin }}\right)$. The pinning variable $\lambda$ is defined on the boundary $\Gamma$ of the droplet. On the unpinned regions, $\lambda$ saturates to $\pm P_{\text {pin. }}$. On the pinned regions $(\mathbf{u} \cdot \boldsymbol{\nu}=0), \lambda$ varies between $-P_{\text {pin }}$ and $P_{\text {pin }}$ and acts as a Lagrange multiplier to enforce the zero normal velocity constraint.

We further modify the boundary condition by adding a 'friction' term [54] which models the 'pinning' effect (see [12, 20, 18, 59, 48, 21, 34]) that occurs at the three-phase contact line of the liquid-gas interface (i.e. the boundary $\Gamma$ ). This is written as

$$
p=\kappa+E+\lambda+D_{\mathrm{visc}} \mathbf{u} \cdot \boldsymbol{\nu}, \quad \lambda \in P_{\mathrm{pin}} \operatorname{sgn}(\mathbf{u} \cdot \boldsymbol{\nu}), \quad \text { on } \Gamma,
$$

where $P_{\text {pin }}$ and $D_{\text {visc }}$ are contact line friction coefficients, $\nu$ is the unit outward normal, and $\lambda$ takes values in the graph of $P_{\text {pin }} \operatorname{sgn}(\mathbf{u} \cdot \boldsymbol{\nu})$ and is defined on the boundary $\Gamma$. See Figure 1 for a droplet diagram with pinned and unpinned regions and more discussion of $\lambda$. Physically, $\lambda$ is a pressure that pushes against the liquid-gas interface to oppose its motion, which is analogous to static (Coulombic) friction. However, the regions of the interface that are pinned/unpinned are not known a priori. This must be determined as part of the solution process. The extra $D_{\text {visc }} \mathbf{u} \cdot \boldsymbol{\nu}$ term acts as a viscous damping effect on the interface motion in the sense that friction pushes against the interface harder with increasing velocity [46], [35], and accounts for contact angle hysteresis effects observed in EWOD devices. Both loss mechanisms are responsible for the excellent space-time match between our simulations and lab experiments [57].

Finally, we need an equation to describe the time-varying motion of the boundary $\Gamma$. Each point $\mathbf{X}$ on $\Gamma$ satisfies the $\operatorname{ODE}\left(\partial_{t} \mathbf{X}-\mathbf{u}(\mathbf{X})\right) \cdot \boldsymbol{\nu}=0$. This leaves us free to use the following equation of motion for the interface:

$$
\partial_{t} \mathbf{X}=\mathbf{u}(\mathbf{X})
$$

which is more convenient for our formulation. Basically, the droplet boundary moves with the velocity of the fluid at the boundary. The normal component of the velocity effectively determines the shape of the droplet whereas the tangential component corresponds to a reparametrization of the parametric boundary (see definition (9) for the interface). 


\section{Time discretization and mixed formulation}

Before stating the variational formulation, we define the time-discrete version of (1) and (2) in strong form. Then we obtain a formal weak formulation of the PDE in (4)-(7) to motivate the functional setting. We end this section by showing the equivalence of the weak and strong formulation for smooth solutions and domains.

\subsection{Time discretization}

We partition the time axis into time intervals $\delta t_{i}$, for $i$ in some finite index set. Let $\Omega^{i}$ and $\Gamma^{i}:=$ $\partial \Omega^{i}$ be the droplet domain and interface at time $t_{i}$, which are assumed known and smooth in the subsequent analysis. We obtain the velocity $\mathbf{u}^{i+1}$ at time $t_{i+1}$ by solving the following semi-implicit time-discrete version of (1)-2):

$$
\begin{aligned}
\alpha \frac{\mathbf{u}^{i+1}-\mathbf{u}^{i}}{\delta t_{i+1}}+\beta \mathbf{u}^{i+1}+\nabla p^{i+1}=0 & \text { in } \Omega^{i}, \\
\nabla \cdot \mathbf{u}^{i+1}=0 & \text { in } \Omega^{i}, \\
p^{i+1}-\kappa^{i+1}-E^{i}-\lambda^{i+1}-D_{\mathrm{visc}} \mathbf{u}^{i+1} \cdot \boldsymbol{\nu}^{i}=0 & \text { on } \Gamma^{i}, \\
\lambda^{i+1} \in P_{\text {pin }} \operatorname{sgn}\left(\mathbf{u}^{i+1} \cdot \nu^{i}\right) & \text { on } \Gamma^{i},
\end{aligned}
$$

where $\mathbf{u}^{i}, \boldsymbol{\nu}^{i}$, and $E^{i}$ are known quantities at time index $t_{i}, \kappa^{i+1}$ is an approximation of the curvature of the boundary at time $t_{i+1}$, and all other terms with superscript $i+1$ are variables to be solved for. Here, we have used an Euler method to approximate the time derivative term $\partial_{t} \mathbf{u}$.

Next, let $\mathbf{x}^{i}(\cdot)$ be the identity map on $\Gamma^{i}$ and let $\mathbf{X}^{i+1}(\cdot): \Gamma^{i} \rightarrow \mathbb{R}^{2}$. The interface position at time $t_{i+1}$ is defined by a time-discrete version of the interface motion (3) as

$$
\mathbf{X}^{i+1}\left(\mathbf{x}^{i}\right):=\mathbf{x}^{i}+\delta t_{i+1} \mathbf{u}^{i+1}\left(\mathbf{x}^{i}\right),
$$

where $\mathbf{u}^{i+1}: \Gamma^{i} \rightarrow \mathbb{R}^{2}$ is the velocity at the next time index. Given $\mathbf{X}^{i+1}$, we define the interface at time $t_{i+1}$ by

$$
\Gamma^{i+1}:=\mathbf{X}^{i+1}\left(\Gamma^{i}\right)
$$

and define the domain $\Omega^{i+1}$ at time $t_{i+1}$ to be the domain enclosed by $\Gamma^{i+1}$.

The solution domain $\overline{\Omega^{i}}$ is kept explicit in (4)-(7) when solving for the new velocity $\mathbf{u}^{i+1}$, which is a linearization step. A higher order method may be built from the above first order semi-implicit method, just as was done in [3].

Calculation of the curvature $\kappa^{i+1}$ is not solely based on $\Gamma^{i}$ (i.e. is not fully explicit). In fact, we have some freedom in choosing how the curvature is computed. We recall some notation from differential geometry. The surface gradient on a manifold $\Gamma$ is denoted by $\nabla_{\Gamma}$ and is a vector operator [19]. When $\nabla_{\Gamma}$ is applied to a vector $\mathbf{r}$ on a 1-D curve $\Gamma$, it becomes $\nabla_{\Gamma} \mathbf{r}=\partial_{s} \mathbf{r} \otimes \tau$, where $\partial_{s}$ is the derivative with respect to arc-length and $\tau$ is the unit tangent vector (oriented with respect to $s$ ). The Laplace-Beltrami operator is defined as $\Delta_{\Gamma}:=\nabla_{\Gamma} \cdot \nabla_{\Gamma}$, and for a 1-D curve is just the second derivative with respect to arc-length. See [23, 19, 54] for more details.

As in [3, 23], we take advantage of the expression for the vector curvature on $\Gamma^{i}$ [19]

$$
\kappa^{i}:=\kappa^{i} \nu^{i}=-\Delta_{\Gamma^{i}} \mathbf{x}^{i}
$$


We multiply [6] by $\nu^{i}$ and view the resulting expression as a natural boundary condition:

$$
p^{i+1} \boldsymbol{\nu}^{i}-\kappa^{i+1} \boldsymbol{\nu}^{i}-\lambda^{i+1} \boldsymbol{\nu}^{i}-D_{\mathrm{visc}}\left(\mathbf{u}^{i+1} \cdot \boldsymbol{\nu}^{i}\right) \boldsymbol{\nu}^{i}=E^{i} \boldsymbol{\nu}^{i} .
$$

Hence, we impose a semi-implicit approximation to $\kappa^{i+1}$ defined by

$$
\kappa^{i+1} \nu^{i}:=-\Delta_{\Gamma^{i}} \mathbf{X}^{i+1}=-\Delta_{\Gamma^{i}} \mathbf{x}^{i}-\delta t_{i+1} \Delta_{\Gamma^{i}} \mathbf{u}^{i+1}=\kappa^{i} \nu^{i}-\delta t_{i+1} \Delta_{\Gamma^{i}} \mathbf{u}^{i+1},
$$

where we used equations $(8)$ and $(10)$. This definition demands an additional condition, namely that

$$
\delta t_{i+1}\left(\Delta_{\Gamma^{i}} \mathbf{u}^{i+1}\right) \cdot \tau^{i}=0,
$$

and is consistent with the fully continuous problem (i.e. take $\delta t_{i+1} \rightarrow 0$ ). Ergo,

$$
\Delta_{\Gamma^{i}} \mathbf{u}^{i+1} \propto \nu^{i} .
$$

Whether this decision is compatible, and thus there is a solution, is not obvious and must be assessed. See Theorem 4.1 for existence in the semi-discrete problem.

\subsection{Formal weak formulation}

Multiplying equations (4) and (5) by smooth test functions $\mathbf{v}$ and $q$, integrating by parts and using equations (11) and (12), we obtain

$$
\begin{aligned}
&\left(\frac{\alpha}{\delta t_{i+1}}+\beta\right) \int_{\Omega^{i}} \mathbf{u}^{i+1} \cdot \mathbf{v}- \int_{\Omega^{i}} p^{i+1} \nabla \\
&+\mathbf{v}+\delta t_{i+1} \int_{\Gamma^{i}} \nabla_{\Gamma^{i}} \mathbf{u}^{i+1}: \nabla_{\Gamma^{i}} \mathbf{v} \\
& \quad+\int_{\Gamma^{i}} \lambda^{i+1} \nu^{i} \cdot \mathbf{v}+D_{\mathrm{visc}} \int_{\Gamma^{i}}\left(\mathbf{u}^{i+1} \cdot \boldsymbol{\nu}^{i}\right)\left(\mathbf{v} \cdot \boldsymbol{\nu}^{i}\right) \\
&=\frac{\alpha}{\delta t_{i+1}} \int_{\Omega^{i}} \mathbf{u}^{i} \cdot \mathbf{v}-\int_{\Gamma^{i}} E^{i} \mathbf{v} \cdot \boldsymbol{\nu}^{i}-\int_{\Gamma^{i}} \nabla_{\Gamma^{i}} \mathbf{x}^{i}: \nabla_{\Gamma^{i}} \mathbf{v}
\end{aligned}
$$

and

$$
\int_{\Omega^{i}} q \nabla \cdot \mathbf{u}^{i+1}=0
$$

Equations (15) and (16) need to be supplemented by equation (7) linking the Lagrange multiplier $\lambda^{i+1}: \Gamma^{i} \rightarrow\left[-P_{\text {pin }}, P_{\text {pin }}\right]$ to $\mathbf{u}^{i+1} \cdot \nu^{i}$. In order to obtain a formulation that fits our variational setting, we introduce the inequality

$$
\int_{\Gamma^{i}}\left(\mu-\lambda^{i+1}\right) \mathbf{u}^{i+1} \cdot \nu^{i} \leqslant 0
$$

for all $\mu: \Gamma^{i} \rightarrow\left[-P_{\text {pin }}, P_{\text {pin }}\right]$ smooth. To see the connection between (7) and (17), consider the interior int $\left(\mathcal{C}_{+}^{i+1}\right)$ of the contact set $\mathcal{C}_{+}^{i+1}$ where $\lambda^{i+1}=P_{\text {pin. }}$. Let $\mu=\lambda^{i+1}-\epsilon \varphi$ for $\varphi \geqslant 0$ smooth with support contained in $\operatorname{int}\left(\mathcal{C}_{+}^{i+1}\right)$ and $\epsilon>0$ sufficiently small. This choice of $\mu$ guarantees that $\mu$ is admissible in (17) and leads to

$$
\int_{\Gamma^{i}} \varphi\left(\mathbf{u}^{i+1} \cdot \boldsymbol{\nu}^{i}\right) \geqslant 0
$$

or $\mathbf{u}^{i+1} \cdot \boldsymbol{\nu}^{i} \geqslant 0 \operatorname{in} \operatorname{int}\left(\mathcal{C}_{+}^{i+1}\right)$. Similarly, we get $\mathbf{u}^{i+1} \cdot \boldsymbol{\nu}^{i} \leqslant 0 \operatorname{in} \operatorname{int}\left(\mathcal{C}_{-}^{i+1}\right)$, i.e. where $\lambda^{i+1}=-P_{\text {pin }}$. On the other hand, in the interior $\operatorname{int}\left(\mathcal{N}^{i+1}\right)$ of the noncoincidence set $\mathcal{N}^{i+1}$, where $-P_{\text {pin }}<\lambda<$ $P_{\text {pin }}$, we have $\mathbf{u}^{i+1} \cdot \boldsymbol{\nu}^{i}=0$ because $\varphi$ can have arbitrary sign with $\mu$ still admissible. Altogether, we conclude that (17) is equivalent to $\lambda^{i+1} \in P_{\text {pin }} \operatorname{sgn}\left(\mathbf{u}^{i+1} \cdot \boldsymbol{\nu}^{i}\right)$, i.e. (7). 


\subsection{Functional setting}

This section determines the correct function space in which to pose the variational form (15)-(17).

3.3.1 Velocity space. We start by considering the space for the velocity. In view of (15) and (16), the velocity $\mathbf{u}^{i+1}$ must be in $H\left(\operatorname{div} ; \Omega^{i}\right)=\left\{\mathbf{v} \in\left[L^{2}\left(\Omega^{i}\right)\right]^{2}: \nabla \cdot \mathbf{v} \in L^{2}\left(\Omega^{i}\right)\right\}$ and $H^{1}\left(\Gamma^{i}\right)$. Ergo, we define the scalar product $s_{i}(\cdot, \cdot): C^{\infty}\left(\overline{\Omega^{i}}\right) \times C^{\infty}\left(\overline{\Omega^{i}}\right) \rightarrow \mathbb{R}$ by

$$
s_{i}(\mathbf{u}, \mathbf{v}):=\int_{\Omega^{i}} \mathbf{u} \cdot \mathbf{v}+\int_{\Omega^{i}}(\nabla \cdot \mathbf{u})(\nabla \cdot \mathbf{v})+\delta t_{i+1}\left(\int_{\Gamma^{i}} \mathbf{u} \cdot \mathbf{v}+\int_{\Gamma^{i}} \nabla_{\Gamma^{i}} \mathbf{u}: \nabla_{\Gamma^{i}} \mathbf{v}\right),
$$

and define a norm induced by this scalar product,

$$
\|\mathbf{u}\|_{\mathbb{V}^{i}}:=\sqrt{s_{i}(\mathbf{u}, \mathbf{u})} .
$$

Next, define the function space $\mathbb{V}^{i}$ as the closure of $C^{\infty}\left(\overline{\Omega^{i}}\right)$ with respect to the norm $\|\cdot\|_{\mathbb{V} i}$; see [36, 37, 25].

Therefore, the space $\mathbb{V}^{i}$ and norm $\|\cdot\|_{\mathbb{V}^{i}}$ define a Hilbert space [25, 36], which is required for the well-posedness of the variational formulation. We now make note of some functional relations that will be useful later. First, using Definition 3.2, Corollary 3.4, and Theorem 3.17 in [1], one can show the following inclusions:

$$
\left[H^{1}\left(\Omega^{i}\right)\right]^{2} \cap\left[H^{1}\left(\Gamma^{i}\right)\right]^{2} \subset \mathbb{V}^{i} \subset H\left(\operatorname{div}, \Omega^{i}\right) \cap\left[H^{1}\left(\Gamma^{i}\right)\right]^{2} .
$$

Now we introduce a more precise characterization of the velocity space $\mathbb{V}^{i}$. Let $\mathbf{v} \in \mathbb{V}^{i}$, which is a function defined on the closure $\overline{\Omega^{i}}$, and let $\mathbf{v}_{\text {int }}$ denote $\mathbf{v}$ on the open set $\Omega^{i}$. Because of a standard result for the normal trace of $H\left(\operatorname{div}, \Omega^{i}\right)$ functions, [29], we know that $\|\mathbf{v} \cdot \boldsymbol{\nu}\|_{-1 / 2, \Gamma^{i}} \leqslant$ $\sqrt{2}\left\|\mathbf{v}_{\text {int }}\right\|_{H\left(\operatorname{div}, \Omega^{i}\right)}$. This means that the normal component of the velocity on the boundary is linked to the velocity in the interior. No such result exists for the tangential component, which is not well defined for functions only in $H\left(\operatorname{div} ; \Omega^{i}\right)$. However, functions in $\mathbb{V}^{i}$ have boundary values in $\left[H^{1}\left(\Gamma^{i}\right)\right]^{2}$. Therefore, the tangential velocity may be disconnected from the interior velocity. So for $\mathbf{v} \in \mathbb{V}^{i}$, we let $\mathbf{v}_{\boldsymbol{\tau}}$ denote the tangential component of $\mathbf{v}$ on $\Gamma^{i}$, which has no connection with $\mathbf{v}_{\text {int }}$. This leads to the following decomposition for $\mathbf{v}$ :

$$
\left.\mathbf{v}\right|_{\Omega^{i}}=\mathbf{v}_{\text {int }},\left.\quad \mathbf{v} \cdot \boldsymbol{\nu}\right|_{\Gamma^{i}}=\mathbf{v}_{\text {int }} \cdot \nu,\left.\quad \mathbf{v} \cdot \boldsymbol{\tau}\right|_{\Gamma^{i}}=\mathbf{v}_{\boldsymbol{\tau}} \cdot \boldsymbol{\tau}
$$

Recalling that $\Gamma^{i}$ is a closed surface, it is more convenient to equip $\mathbb{V}^{i}$ with the norm

$$
\|\mathbf{u}\|_{\mathbb{V}^{i}}^{2}:=\int_{\Omega^{i}}|\mathbf{u}|^{2}+\int_{\Omega^{i}}|\nabla \cdot \mathbf{u}|^{2}+\delta t_{i+1} \int_{\Gamma^{i}}\left|\nabla_{\Gamma} \mathbf{u}\right|^{2} .
$$

The triple norm $\||\cdot|\|_{\mathbb{V} i}$ is equivalent to $\|\cdot\|_{\mathbb{V} i}$ as stated in the following lemma. Note the result is not obvious due to the nonstandard space $\mathbb{V}^{i}$ characterized by 21].

LEMMA 3.1 (Alternative norm on $\mathbb{V}$ ) Let $\Gamma^{i}$ be Lipschitz. Then $\|\cdot\|_{\mathbb{V} i}$ is a norm on $\mathbb{V}^{i}$ equivalent to $\|\cdot\|_{\mathbb{V}^{i}}$.

Proof. For convenience, we drop the time index notation ' $i$ '. The only norm property to check is that $\|\mid \mathbf{u}\| \|_{\mathbb{V}}=0$ implies $\mathbb{V} \ni \mathbf{u}=0$. So, assume $\mathbf{u} \in \mathbb{V}$, and let $\|\mathbf{u}\| \|_{\mathbb{V}}=0$. Then $\left\|\nabla_{\Gamma} \mathbf{u}\right\|_{0, \Gamma}=0$, 
which implies $\left.\mathbf{u}\right|_{\Gamma}=\mathbf{u}_{0}$ (constant vector) in $L^{2}(\Gamma)$. By standard Sobolev embedding [40], [1], $\mathbf{u} \cdot \boldsymbol{\nu}=\mathbf{u}_{0} \cdot \boldsymbol{\nu}$ in $L^{2}(\Gamma) \subset H^{-1 / 2}(\Gamma)$. Ergo, we have

$$
0=\sqrt{2}\|\mathbf{u}\|_{H(\operatorname{div}, \Omega)} \geqslant\|\mathbf{u} \cdot \boldsymbol{\nu}\|_{-1 / 2, \Gamma}=\left\|\mathbf{u}_{0} \cdot \boldsymbol{\nu}\right\|_{-1 / 2, \Gamma},
$$

which implies that $\mathbf{u}_{0} \cdot \boldsymbol{\nu}=0$ a.e. on $\Gamma$. Hence, $\mathbf{u}_{0}=0$ and $\mathbf{u}=0$ in $\mathbb{V}$. So, $\|\cdot\| \mathbb{V}$ is a norm on $\mathbb{V}$. Specifically, $\|\cdot\| \| \mathbb{V}$ is equivalent to the $\mathbb{V}$ norm defined in $(19)$, i.e.

$$
\|\mathbf{u}\|_{\mathbb{V}} \geqslant\|\mathbf{u}\|_{\mathbb{V}} \geqslant c_{\mathbb{V}}\|\mathbf{u}\|_{\mathbb{V}}
$$

where $c \mathbb{V}>0$ is a constant that only depends on $\Omega$. The first inequality is trivial; the second follows from a classical compactness argument (see for instance [1, 25]).

3.3.2 The pressure space. The pressure space $\left(\right.$ denoted $\left.\mathbb{Q}^{i}\right)$ is defined by

$$
\mathbb{Q}^{i}:=L^{2}\left(\Omega^{i}\right)
$$

and we denote $\mathbb{Q}_{0}^{i}:=\left\{q \in \mathbb{Q}^{i}: \int_{\Omega^{i}} q=0\right\}$, i.e. $L^{2}$ functions with mean value zero. The norm for $\mathbb{Q}^{i}$ is denoted $\|\cdot\|_{\mathbb{Q}^{i}}:=\|\cdot\|_{0, \Omega^{i}}$.

3.3.3 The boundary multiplier space. The space for the boundary Lagrange multiplier $\lambda^{i+1}$ (denoted $\mathbb{M}^{i}$ ) is a dual space and is defined in the following way. For $\mathbf{v} \in H^{1}\left(\Gamma^{i}\right)^{2}$ and $\Gamma^{i}$ smooth, the product $\mathbf{v} \cdot \boldsymbol{\nu}^{i}$ belongs to $H^{1}\left(\Gamma^{i}\right)$. Hence,

$$
\mathbb{G}^{i}:=H^{1}\left(\Gamma^{i}\right) .
$$

Next, define $\mathbb{M}^{i}$ as the dual of $\mathbb{G}^{i}[25]$, [37], i.e. $\mathbb{M}^{i}:=\left(H^{1}\left(\Gamma^{i}\right)\right)^{*}$, and let $\langle\cdot, \cdot\rangle$ denote the duality pairing between $\mathbb{G}^{i}$ and $\mathbb{M}^{i}$. The norm on $\mathbb{M}^{i}$ is then given by

$$
\|\mu\|_{\mathbb{M}^{i}}=\sup _{\eta \in \mathbb{G}^{i}, \eta \neq 0} \frac{\langle\mu, \eta\rangle}{\|\eta\|_{\mathbb{G}^{i}}} .
$$

In addition, $\lambda^{i+1}$ must be restricted to values between $-P_{\text {pin }}$ and $P_{\text {pin. }}$. Therefore, we introduce $\Lambda^{i}$, the closed convex set contained in $\mathbb{M}^{i}$ defined by

$$
\Lambda^{i}:=\left\{\mu \in \mathbb{M}^{i}:|\langle\mu, \eta\rangle| \leqslant P_{\mathrm{pin}}\langle 1, \eta\rangle=P_{\mathrm{pin}} \int_{\Gamma^{i}} \eta, \forall \eta \in \mathbb{G}^{i}, \eta \geqslant 0 \text { a.e. }\right\} .
$$

The next crucial lemma follows from 27.

LEmma 3.2 (Characterization of $\Lambda^{i}$ ) $\Lambda^{i} \subset \mathbb{M}^{i}$ is a bounded convex set. In particular, the elements $\mu$ of $\Lambda^{i}$ are functions in $L^{\infty}\left(\Gamma^{i}\right)$ and satisfy $|\mu(\mathbf{x})| \leqslant P_{\text {pin }}$, a.e. $\mathbf{x} \in \Gamma^{i}$.

Proof. We are going to show that functionals in this convex set are bounded, i.e. $\mu \in \Lambda^{i} \Rightarrow \mu \in$ $L^{\infty}\left(\Gamma^{i}\right)$. Let $\rho_{\epsilon}$ be a smooth mollifier defined on $\Gamma^{i}$. Then $\rho_{\epsilon} \in \mathbb{G}^{i}$. Define a smoothed version of $\mu \in \Lambda^{i}$ by

$$
\mu_{\epsilon}(s):=\left\langle\mu, \rho_{\epsilon}(s-\cdot)\right\rangle, \quad \text { so that } \quad\left|\mu_{\epsilon}(s)\right| \leqslant P_{\text {pin }} \int_{\Gamma^{i}} \rho_{\epsilon}(s-t) \mathrm{d} t=P_{\text {pin }} .
$$


By density, $\mu_{\epsilon} \rightarrow \mu \in \mathbb{M}^{i}$ as $\epsilon \rightarrow 0$. By the definition of the $\mathbb{M}^{i}$ norm 26, this implies

$$
\left\langle\mu_{\epsilon}-\mu, \eta\right\rangle \rightarrow 0 \quad \text { for all } \eta \in \mathbb{G}^{i}
$$

Next, let $\psi^{+} \in L^{1}\left(\Gamma^{i}\right)$ be such that $\psi^{+} \geqslant 0$ a.e. Then we have

$$
\begin{aligned}
& \int_{\Gamma^{i}} \mu_{\epsilon}(s) \psi^{+}(s) \mathrm{d} s=\int_{\Gamma^{i}}\left\langle\mu, \rho_{\epsilon}(s-\cdot)\right\rangle \psi^{+}(s) \mathrm{d} s=\int_{\Gamma^{i}}\left\langle\mu, \psi^{+}(s) \rho_{\epsilon}(s-\cdot)\right\rangle \mathrm{d} s \\
&=\left\langle\mu, \int_{\Gamma^{i}} \psi^{+}(s) \rho_{\epsilon}(s-\cdot) \mathrm{d} s\right\rangle=:\left\langle\mu, \psi_{\epsilon}^{+}\right\rangle \leqslant P_{\text {pin }} \int_{\Gamma^{i}} \psi_{\epsilon}^{+}<\infty \quad \text { for all } \epsilon,
\end{aligned}
$$

where we interchanged the order of integration in the second line. Note that $\psi_{\epsilon}^{+} \in \mathbb{G}^{i}$ by the same argument as for $\rho_{\epsilon}$. For a general $\psi$, we can split it into its positive and negative parts, take the absolute value, and use the triangle inequality to get

$$
\left|\int_{\Gamma^{i}} \mu_{\epsilon} \psi \mathrm{d} s\right|<\infty
$$

for all $\psi \in L^{1}\left(\Gamma^{i}\right)$ and all $\epsilon>0$. Therefore, $\mu_{\epsilon}$ converges weak-* to a functional $\omega \in L^{1}\left(\Gamma^{i}\right)^{*}=$ $L^{\infty}\left(\Gamma^{i}\right)$, i.e.

$$
\left\langle\mu_{\epsilon}-\omega, \psi\right\rangle \rightarrow 0 \quad \text { for all } \psi \in L^{1}\left(\Gamma^{i}\right) .
$$

Since $\mathbb{G}^{i} \subset L^{1}\left(\Gamma^{i}\right)$, using 29 , we find that $\mu_{\epsilon} \rightarrow \mu=\omega \in L^{\infty}\left(\Gamma^{i}\right)$. Note that $\mu \in L^{2}\left(\Gamma^{i}\right)$ because $\Gamma^{i}$ has bounded measure.

\subsection{Equivalence of weak and strong forms}

We show that the weak formulation implies the strong form assuming the solution is smooth and the domain is smooth. Normally this is obvious, but in our case there is an 'artifact' of the weak formulation that arises because of the definition of $\mathbb{V}^{i}$. The space $\mathbb{V}^{i}$ contains vector velocity functions whose tangential component on $\Gamma^{i}$ is unrelated to the vector function values in the interior of $\Omega^{i}$. This could affect the equivalence with the time-discrete strong form (given earlier) if the tangential component of the solution is completely arbitrary. We now clarify this issue by showing that the tangential velocity is controlled by the normal velocity in the $H^{1}\left(\Gamma^{i}\right)$ norm, followed by a consistency result.

3.4.1 Control of the tangential velocity. The strong form equations in the bulk can be derived from the weak form in the standard way (i.e. take a test function with compact support in $\Omega^{i}$ ), so we concentrate on deriving the strong form of the pressure boundary condition. After integrating the pressure term by parts in (15), applying the strong form of the momentum equation, integrating the $\nabla_{\Gamma^{i}} \mathbf{x}^{i}: \nabla_{\Gamma^{i}} \mathbf{v}$ term by parts and recalling the definition of curvature (10), we get

$$
\begin{aligned}
-\int_{\Gamma^{i}} p^{i+1} \mathbf{v} \cdot \boldsymbol{\nu}^{i}+\delta & t_{i+1} \int_{\Gamma^{i}} \nabla_{\Gamma^{i}} \mathbf{u}^{i+1}: \nabla_{\Gamma^{i}} \mathbf{v}+\int_{\Gamma^{i}} \lambda^{i+1} \mathbf{v} \cdot \boldsymbol{\nu}^{i} \\
& +D_{\mathrm{visc}} \int_{\Gamma^{i}}\left(\mathbf{u}^{i+1} \cdot \boldsymbol{\nu}^{i}\right)\left(\mathbf{v} \cdot \boldsymbol{\nu}^{i}\right)=-\int_{\Gamma^{i}} E^{i} \mathbf{v} \cdot \boldsymbol{\nu}^{i}-\int_{\Gamma^{i}} \kappa^{i} \boldsymbol{\nu}^{i} \cdot \mathbf{v} .
\end{aligned}
$$


Only the normal component of $\mathbf{v}$ appears in (33), except in the $\delta t_{i+1}$ term. Ergo, we replace $\mathbf{v}$ by $\left(\mathbf{v}_{\text {int }} \cdot \boldsymbol{\nu}^{i}\right) \boldsymbol{\nu}^{i}$ where appropriate. Writing $\mathbf{v}$ (in the remaining $\delta t_{i+1}$ integral) in terms of the decoupled normal and tangential components (21), both of which are assumed to be smooth and arbitrary, leads to

$$
\begin{aligned}
0= & -\int_{\Gamma^{i}} p^{i+1} \mathbf{v}_{\mathrm{int}} \cdot \boldsymbol{\nu}^{i}+\int_{\Gamma^{i}} \lambda^{i+1} \mathbf{v}_{\mathrm{int}} \cdot \boldsymbol{\nu}^{i}+\int_{\Gamma^{i}} E^{i} \mathbf{v}_{\mathrm{int}} \cdot \boldsymbol{\nu}^{i} \\
& +\int_{\Gamma^{i}} \kappa^{i} \boldsymbol{\nu}^{i} \cdot \mathbf{v}_{\mathrm{int}}+D_{\mathrm{visc}} \int_{\Gamma^{i}}\left(\mathbf{u}^{i+1} \cdot \boldsymbol{\nu}^{i}\right)\left(\mathbf{v}_{\mathrm{int}} \cdot \boldsymbol{\nu}^{i}\right) \\
& +\delta t_{i+1} \int_{\Gamma^{i}} \nabla_{\Gamma^{i}} \mathbf{u}^{i+1}: \nabla_{\Gamma^{i}}\left(\left(\mathbf{v}_{\mathrm{int}} \cdot \boldsymbol{\nu}^{i}\right) \boldsymbol{\nu}^{i}+\mathbf{v}_{\boldsymbol{\tau}}\right) .
\end{aligned}
$$

Next, we choose $\mathbf{v}_{\text {int }}=0$, expand $\mathbf{u}^{i+1}$ in terms of its decoupled components and apply the CauchySchwarz inequality, to get $\left\|\nabla_{\Gamma^{i}} \mathbf{u}_{\boldsymbol{\tau}}^{i+1}\right\|_{0, \Gamma^{i}} \leqslant\left\|\nabla_{\Gamma^{i}}\left(\left(\mathbf{u}_{\text {int }}^{i+1} \cdot \boldsymbol{\nu}^{i}\right) \boldsymbol{\nu}^{i}\right)\right\|_{0, \Gamma^{i}}$. The following lemma gives control of the full $H^{1}\left(\Gamma^{i}\right)$ norm:

LEMma 3.3 (Poincaré for tangential field) Let $\Gamma^{i}$ be a 1-D closed curve of class $W_{\infty}^{2}$ and $\mathbf{v} \in$ $H^{1}\left(\Gamma^{i}\right)$ such that $\mathbf{v} \cdot \boldsymbol{\nu}=0$, i.e. $\mathbf{v}$ is tangential on $\Gamma^{i}$. Then

$$
\|\mathbf{v}\|_{0, \Gamma^{i}} \leqslant C\left\|\nabla_{\Gamma} \mathbf{v}\right\|_{0, \Gamma^{i}}
$$

where $C$ is an independent constant.

Proof. The proof follows by similar reasoning to Lemma 3.1. If $\nabla_{\Gamma} \mathbf{v}=\partial_{s} \mathbf{v} \otimes \boldsymbol{\tau}=0$, then $\mathbf{v}$ is constant on $\Gamma^{i}$. But $\mathbf{v}$ is tangential, so $\mathbf{v}=0$. A classical compactness argument completes the proof (see [1, 25]). A more direct proof (see [54]) indicates that the constant $C$ only depends on the diameter of a ball containing $\Gamma^{i}$.

Thus, we have

$$
\left\|\mathbf{u}_{\boldsymbol{\tau}}^{i+1}\right\|_{1, \Gamma^{i}} \leqslant C\left\|\nabla_{\Gamma^{i}}\left(\left(\mathbf{u}_{\mathrm{int}}^{i+1} \cdot \boldsymbol{\nu}^{i}\right) \boldsymbol{\nu}^{i}\right)\right\|_{0, \Gamma^{i}} .
$$

This means that the decoupled tangential component is not completely arbitrary and is controlled by the normal component of the velocity (in $H^{1}\left(\Gamma^{i}\right)$ ), which is linked with the interior velocity.

3.4.2 Consistency between interior and boundary velocity for strong solution. Suppose we have a smooth strong solution $\left(\mathbf{u}^{i+1}, p^{i+1}, \lambda^{i+1}\right)$ on $\overline{\Omega^{i}}$ of the PDE (4)-(7). This implies that $\mathbf{u}_{\boldsymbol{\tau}}^{i+1} \cdot \boldsymbol{\tau}$ is the tangential trace of $\mathbf{u}_{\text {int }}^{i+1}$ in $\Omega$. Moreover, $\left(\mathbf{u}^{i+1}, p^{i+1}, \lambda^{i+1}\right)$ is also a weak solution by the same derivation we gave in Section 3.2 and is unique by Lemma 4.5 . Thus, the tangential velocity on the boundary and the velocity in the interior are always linked in the case of a strong solution.

This implies that the full velocity $\mathbf{u}^{i+1}$ on $\Gamma^{i}$ is well defined for updating the interface in the case of strong solution. Note that for the fully discrete method $\mathbf{u}_{\text {int }}^{i+1}$ and $\mathbf{u}_{\boldsymbol{\tau}}^{i+1}$ always coincide because of the definition of our discrete velocity space (see 677). So again, updating the interface is well defined.

3.4.3 Pressure boundary condition. By the previous section, the full velocity $\mathbf{u}^{i+1}$ on $\Gamma^{i}$ is well defined for updating the interface (i.e. moving $\Gamma^{i}$ to $\Gamma^{i+1}$ ) with equation (8). Hence, we can apply (12), which comes from (8), to get the strong form of the boundary condition for the time-discrete problem. We proceed by first rearranging (34), setting $\mathbf{v}_{\boldsymbol{\tau}}=0$, integrating the surface gradient term 
by parts, and plugging in the vector curvature update (12) to obtain

$$
\int_{\Gamma^{i}}\left(-p^{i+1}+\lambda^{i+1}+D_{\mathrm{visc}}\left(\mathbf{u}^{i+1} \cdot \boldsymbol{\nu}^{i}\right)+E^{i}+\kappa^{i+1}\right) \mathbf{v}_{\mathrm{int}} \cdot \boldsymbol{\nu}^{i}=0 .
$$

Choosing $\mathbf{v}_{\text {int }}$ such that $\left.\mathbf{v}_{\text {int }}\right|_{\Gamma^{i}}=\varphi \boldsymbol{\nu}^{i}$ on the boundary for all smooth $\varphi$, we get the strong form of the pressure boundary condition (6). Therefore, we have shown that the weak and strong forms of the time-discrete problem are equivalent.

\subsection{Mixed formulation}

We are now able to define a weak formulation of (4)-(7). We define the following bilinear and linear forms (for convenience of notation):

$$
\begin{aligned}
a^{i}(\mathbf{u}, \mathbf{v})= & \left(\frac{\alpha}{\delta t}+\beta\right)\left(\int_{\Omega^{i}} \mathbf{u} \cdot \mathbf{v}+\gamma \int_{\Omega^{i}}(\nabla \cdot \mathbf{u})(\nabla \cdot \mathbf{v})\right) \\
& +D_{\mathbf{v i s c}} \int_{\Gamma^{i}}\left(\mathbf{u} \cdot \boldsymbol{\nu}^{i}\right)\left(\mathbf{v} \cdot \boldsymbol{\nu}^{i}\right)+\delta t \int_{\Gamma^{i}} \nabla_{\Gamma^{i}} \mathbf{u}: \nabla_{\Gamma^{i}} \mathbf{v}, \\
b^{i}(\mathbf{v}, q)= & -\int_{\Omega^{i}} q \nabla \cdot \mathbf{v}, \quad c^{i}(\mathbf{v}, \mu)=\int_{\Gamma^{i}} \mu \mathbf{v} \cdot \boldsymbol{\nu}^{i}, \\
\chi^{i}(\mathbf{v})= & \frac{\alpha}{\delta t} \int_{\Omega^{i}} \mathbf{u}^{i} \cdot \mathbf{v}-\int_{\Gamma^{i}} E^{i} \mathbf{v} \cdot \boldsymbol{\nu}^{i}-\int_{\Gamma^{i}} \nabla_{\Gamma^{i}} \mathbf{x}^{i}: \nabla_{\Gamma^{i}} \mathbf{v},
\end{aligned}
$$

where we have added an augmented Lagrangian term $\int_{\Omega^{i}}(\nabla \cdot \mathbf{u})(\nabla \cdot \mathbf{v})$, with parameter $1>\gamma>0$, to the first bilinear form $a^{i}(\cdot, \cdot)$. This is consistent with the governing PDE (5) because the velocity is divergence free and is added to improve the convergence of our algorithm for solving the variational inequality (discussed later in Section 6. Note that the coercivity of $a^{i}(\cdot, \cdot)$ on $\left(\mathbb{V}^{i},\|\| \cdot \|_{\mathbb{V}^{i}}\right)$ is immediate from the definition of the velocity space

$$
a^{i}(\mathbf{u}, \mathbf{u}) \geqslant \min \left(\left(\frac{\alpha}{\delta t}+\beta\right) \gamma, 1\right)\||\mathbf{u}|\|_{\mathbb{V}^{i}}^{2}=: c_{a}\left|\|\mathbf{u} \mid\|_{\mathbb{V}^{i}}^{2} .\right.
$$

Typically, the constants $\alpha$ and $\beta$ are larger than 1 . However, note the loss of $H^{1}\left(\Gamma^{i}\right)$ coercivity when the time step $\delta t$ tends to 0 . The EWOD problem is now stated as:

Problem 3.4 (Time-discrete EWOD with pinning) Let $\Omega^{i}$ be a smooth domain. Let $\chi^{i} \in\left(\mathbb{V}^{i}\right)^{*}$ be given data. Then we say that $\mathbf{u}^{i+1} \in \mathbb{V}^{i}, p^{i+1} \in \mathbb{Q}^{i}$, and $\lambda^{i+1} \in \Lambda^{i}$ is a solution of the nonlinear problem if

$$
\begin{aligned}
& a^{i}\left(\mathbf{u}^{i+1}, \mathbf{v}\right)+b^{i}\left(\mathbf{v}, p^{i+1}\right)+c^{i}\left(\mathbf{v}, \lambda^{i+1}\right)=\chi^{i}(\mathbf{v}), \\
& b^{i}\left(\mathbf{u}^{i+1}, q\right)=0, \quad c^{i}\left(\mathbf{u}^{i+1}, \mu-\lambda^{i+1}\right) \leqslant 0,
\end{aligned}
$$

for all $\mathbf{v} \in \mathbb{V}^{i}, q \in \mathbb{Q}^{i}$ and $\mu \in \Lambda^{i}$

\subsection{Simulation of EWOD with pinning}

In Figure 2, we show an illustrative simulation of EWOD driven droplet motion evolving in time. This simulation shows how different parts of the droplet boundary can become pinned and unpinned, depending on the applied voltage. See [56, 54, 57] for more details of the model, simulations and comparisons with lab experiments regarding both shape and dynamics. 

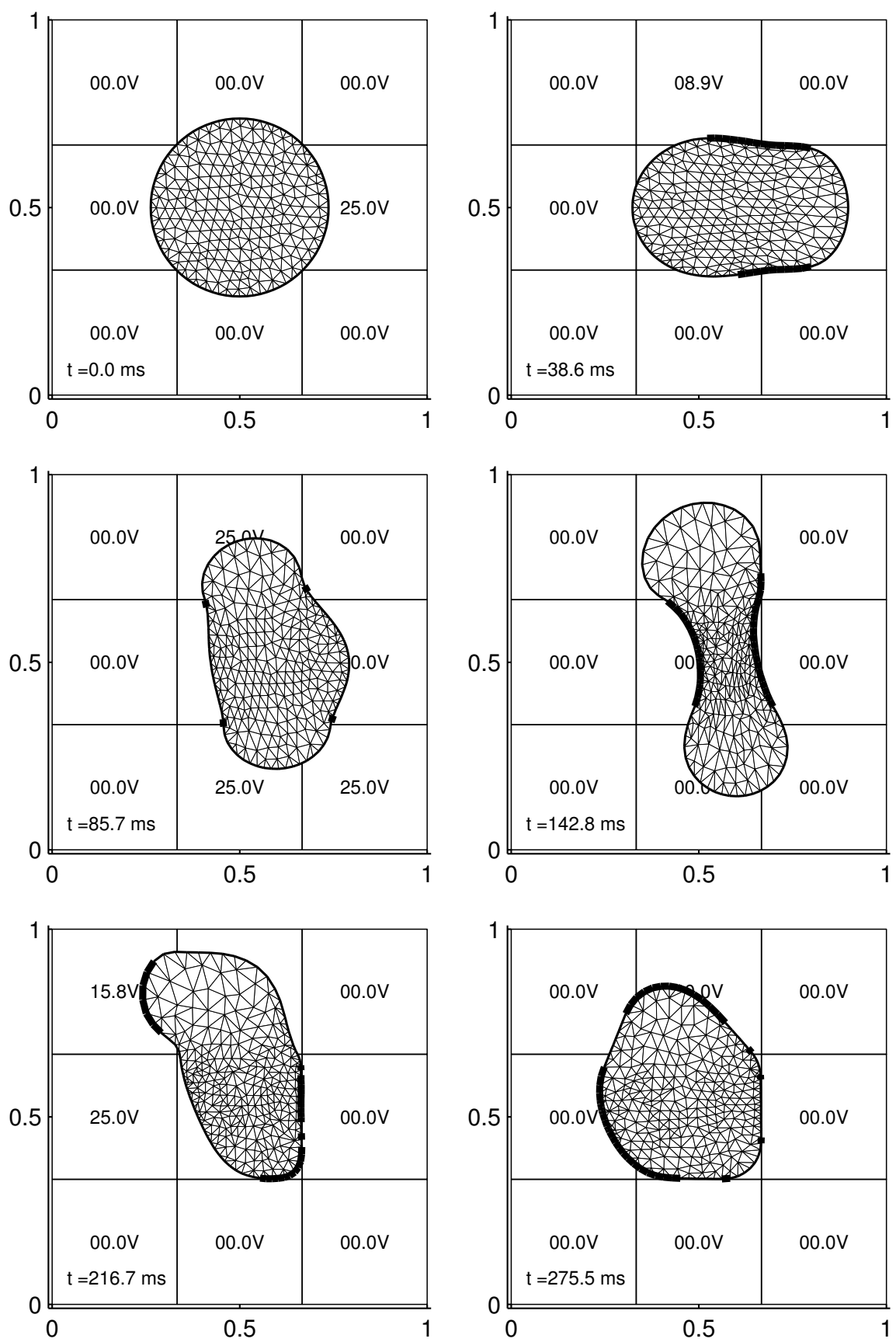

FIG. 2. An EWOD droplet in motion. We show six frames from a time evolving simulation of a droplet actuated by EWOD on a $3 \times 3$ grid of electrodes. The voltage of each electrode pad is displayed. The bold parts of the droplet boundary correspond to where $|\lambda|<P_{\text {pin }}$, i.e. the interface is pinned or $\mathbf{u} \cdot \boldsymbol{\nu}=0$. Notice that in some of the frames, $\mathbf{u} \cdot \boldsymbol{\nu}$ changes from zero to nonzero over a single edge element. 


\section{Well-posedness of time-discrete EWOD with pinning}

We prove in this section the existence of a solution $\left(\mathbf{u}^{i+1}, p^{i+1}, \lambda^{i+1}\right) \in \mathbb{V}^{i} \times \mathbb{Q}^{i} \times \mathbb{M}^{i}$ of Problem 3.4. However, depending on whether the solution is completely pinned (i.e. $|\lambda|<P_{\text {pin }}$ ) or not, the solution may not be unique (see Section 4.1). Therefore, following [41], we obtain a well-posed problem after adding a process that selects the solution with $\lambda$ of minimal norm. Then we obtain an additional regularity for the multiplier $\lambda^{i+1}$ relying on the boundedness of the convex set $\Lambda^{i}$ (Lemma 3.2). We end this section by motivating the maximal expected regularity for the solution of Problem 3.4 For convenience, we denote $\mathbf{u}^{\text {old }}:=\mathbf{u}^{i} \in \mathbb{V}^{i}$ and hereafter drop the time index notation ' $i$ '.

\subsection{Lack of uniqueness}

To understand the inherent lack of uniqueness in the problem, recall the boundary conditions in Section 2.2 (i.e. equation (2)). One can see that to any solution $(\mathbf{u}, p, \lambda)$ there corresponds an infinite number of solutions $(\mathbf{u}, p+C, \lambda+C)$ where $C$ is any constant, provided $\lambda+C \in \Lambda$. This lack of uniqueness can be controlled by enforcing the mean value on the pressure or on $\lambda$. However, it is not possible to fix the mean value of $p$ or $\lambda$ a priori because the boundedness of $\Lambda$ places upper and lower bounds on what the mean value of $\lambda$ (and $p$ ) can be. Also, if the solution satisfies $\lambda=P_{\text {pin }}$ on $\Gamma^{+}$and $\lambda=-P_{\text {pin }}$ on $\Gamma^{-}$, where $\Gamma^{+}$and $\Gamma^{-}$are subsets of the boundary $\Gamma$ with positive measure, then we cannot add a nonzero constant to $\lambda$ and still have an admissible solution; i.e. $\lambda+C$ would not be in $\Lambda$ for $C \neq 0$ (see (27) and (60)).

\subsection{Existence and uniqueness}

To ensure uniqueness, we resort to an idea developed by Lions and Stampacchia [41] and add another inequality that selects, from all possible solutions, the one with $\lambda$ of minimal $\mathbb{M}$ norm. In view of this, we define the convex set $\mathcal{S}$ (possibly empty) of all solutions of Problem 3.4

$$
\mathcal{S}:=\{(\mathbf{u}, p, \lambda) \in \mathbb{V} \times \mathbb{Q} \times \Lambda:(\mathbf{u}, p, \lambda) \text { is a solution of Problem 3.4 }\} .
$$

We recall that $\mathbb{M}$ is a Hilbert space with inner product $\langle\cdot, \cdot\rangle_{\mathbb{M}}$ and now state the main result of this section proved in Sections 4.2.1 and 4.2.2.

THEOREM 4.1 (Existence and uniqueness) Let $\chi \in \mathbb{V}^{*}$ be given data. Then there exists a unique solution $(\mathbf{u}, p, \lambda) \in \mathbb{V} \times \mathbb{Q} \times \Lambda$ of Problem 3.4 that satisfies the additional condition

$$
\langle\lambda, \mu-\lambda\rangle_{\mathbb{M}} \geqslant 0 \quad \text { for all }(\mathbf{v}, q, \mu) \in \mathcal{S} .
$$

REMARK 4.2 (Solution with minimal norm) Condition (41) reflects that $\lambda$ is the $\mathbb{M}$-projection of 0 onto the convex set of solutions $\mathcal{S}$. In other words, $(\mathbf{u}, p, \lambda)$ is the element in $\mathcal{S}$ with $\lambda$ of minimal $\mathbb{M}$ norm.

REMARK 4.3 (Partial inf-sup condition) In a linear saddle point framework (variational inequality replaced by equality), the coercivity of the bilinear form $a(\cdot, \cdot)$ on $\mathbb{V} \times \mathbb{V}$ and the inf-sup condition for $b(\cdot, \cdot)$ and $c(\cdot, \cdot)$ together guarantee the existence and uniqueness of a solution [10]. In the present case, only the partial inf-sup for $b(\cdot, \cdot)$ is needed. For the existence, we employ the boundedness of the convex set $\Lambda$ (see Subsection 4.2.1) whereas the uniqueness is obtained via (41) (see Subsection 4.2.2. 
We follow the ideas proposed by Lions and Stampacchia [41, Theorem 3.2 and 4.1] with the difference that the formulation (40) has a saddle point structure; see also [51, 11]. Therefore, we consider the modified problem

$$
\begin{aligned}
A_{\epsilon}\left(\left(\mathbf{u}_{\epsilon}, \lambda_{\epsilon}\right),\left(\mathbf{u}_{\epsilon}-\mathbf{v}, \lambda_{\epsilon}-\mu\right)\right)+b\left(\mathbf{u}_{\epsilon}-\mathbf{v}, p_{\epsilon}\right)-b\left(\mathbf{u}_{\epsilon}, q\right) \\
\\
\quad \leqslant \chi\left(\mathbf{u}_{\epsilon}-\mathbf{v}\right), \quad \forall(\mathbf{v}, q, \mu) \in \mathbb{V} \times \mathbb{Q} \times \Lambda,
\end{aligned}
$$

where $A_{\epsilon}(\cdot, \cdot)$ is defined for $\epsilon \geqslant 0$ by

$$
A_{\epsilon}((\mathbf{u}, \lambda),(\mathbf{v}, \mu)):=a(\mathbf{u}, \mathbf{v})+\epsilon\langle\lambda, \mu\rangle_{\mathbb{M}}+c(\mathbf{v}, \lambda)-c(\mathbf{u}, \mu) .
$$

Noting that $\mathbb{V}$ is a subspace, the formulation (42) is equivalent to $(40)$ for $\epsilon=0$. Our aim is to prove that for any $\epsilon>0$, there exists a solution $\left(\mathbf{u}_{\epsilon}, p_{\epsilon}, \lambda_{\epsilon}\right) \in \mathbb{V} \times \mathbb{Q} \times \Lambda$ of (42) and the unique solution of Problem 3.4 is given by $\lim _{\epsilon \rightarrow 0}\left(\mathbf{u}_{\epsilon}, p_{\epsilon}, \lambda_{\epsilon}\right)$.

The well-posedness of the solution of 42$)(\epsilon>0)$ is ensured by a classical argument on saddle point problems. Indeed, the coercivity 39] directly implies

$$
c_{a}\|\mathbf{v}\|_{\mathbb{V}}^{2}+\epsilon\|\mu\|_{\mathbb{M}}^{2} \leqslant A_{\epsilon}((\mathbf{v}, \mu),(\mathbf{v}, \mu)),
$$

and the surjectivity of the divergence operator from $H^{1}(\Omega)$ to $L^{2}(\Omega)$ [28, 52] leads to the existence of a constant $C>0$ satisfying

$$
\sup _{\mathbf{v} \in \mathbb{V}} \frac{b(\mathbf{v}, q)}{\|\mathbf{v}\|_{\mathbb{V}}} \geqslant C\|q\|_{\mathbb{Q}} \quad \text { for all } q \in \mathbb{Q} .
$$

Note that 45 follows directly from Lemma 4.6, which is proved independently of the present discussion. Existence of a solution to Problem 3.4 follows next; uniqueness is given in Section 4.2.2.

4.2.1 Existence. Existence without a full inf-sup condition for $p$ and $\lambda$ is guaranteed by the boundedness of the convex set $\Lambda \subset \mathbb{M}$ (Lemma 3.2) and the partial inf-sup (45).

LEMMA 4.4 (Existence) The convex set $\mathcal{S}$ is nonempty.

Proof. We follow Theorem 4.1 in Lions-Stampacchia [41] but in a saddle point framework. Existence of $\left(\mathbf{u}_{\epsilon}, p_{\epsilon}, \lambda_{\epsilon}\right)$ is classical [11, 51]. We first prove that $\left(\mathbf{u}_{\epsilon}, p_{\epsilon}, \lambda_{\epsilon}\right) \in \mathbb{V} \times \mathbb{Q} \times \Lambda$ is uniformly bounded in $\epsilon$. The boundedness of the convex set $\Lambda \subset \mathbb{M}$ (Lemma 3.2) yields

$$
\left\|\lambda_{\epsilon}\right\|_{\mathbb{M}} \preceq 1 .
$$

Moreover, relation (42) implies

$$
A_{\epsilon}\left(\left(\mathbf{u}_{\epsilon}, \lambda_{\epsilon}\right),\left(\mathbf{u}_{\epsilon}, \lambda_{\epsilon}\right)\right) \leqslant b\left(\mathbf{v}-\mathbf{u}_{\epsilon}, p_{\epsilon}\right)+b\left(\mathbf{u}_{\epsilon}, q\right)+A_{\epsilon}\left(\left(\mathbf{u}_{\epsilon}, \lambda_{\epsilon}\right),(\mathbf{v}, \mu)\right)-\chi\left(\mathbf{v}-\mathbf{u}_{\epsilon}\right) .
$$

Choosing $\mathbf{v}=0, \mu=0$, and $q=p_{\epsilon}$ above, using the partial coercivity (44) and the assumption $\chi \in \mathbb{V}^{*}$, leads to the bound for the velocity:

$$
c_{a}\left\|\mathbf{u}_{\epsilon}\right\|_{\mathbb{V}}^{2}+\epsilon\left\|\lambda_{\epsilon}\right\|_{\mathbb{M}}^{2} \preceq 1 .
$$


The uniform bound for $p_{\epsilon}$ is obtained via the inf-sup condition (45), relation (42) and the continuity of $A_{\epsilon}: \mathbb{V} \times \mathbb{M} \times \mathbb{V} \times \mathbb{M} \rightarrow \mathbb{R}:$

$$
\left\|p_{\epsilon}\right\|_{\mathbb{Q}} \preceq \sup _{\mathbf{v} \in \mathbb{V}} \frac{b\left(\mathbf{v}, p_{\epsilon}\right)}{\|\mathbf{v}\|_{\mathbb{V}}} \preceq\left\|\mathbf{u}_{\epsilon}\right\|_{\mathbb{V}}+\left\|\lambda_{\epsilon}\right\|_{\mathbb{M}} \preceq 1
$$

where the bounds (46) and 47) have been used in the last step. The bounds (46), 47), and (48) imply the existence of $(\mathbf{w}, r, \xi) \in \mathbb{V} \times \mathbb{Q} \times \Lambda$ and a subsequence $\left\{\left(\mathbf{u}_{\zeta}, p_{\zeta}, \lambda_{\zeta}\right)\right\}_{\zeta \geqslant 0} \subset\left\{\left(\mathbf{u}_{\epsilon}, p_{\epsilon}, \lambda_{\epsilon}\right)\right\}_{\epsilon} \geqslant 0$ satisfying $\left(\mathbf{u}_{\zeta}, p_{\zeta}, \lambda_{\zeta}\right) \rightarrow(\mathbf{w}, r, \xi)$ in $\mathbb{V} \times \mathbb{Q} \times \Lambda$.

It remains to show that $(\mathbf{w}, r, \xi)$ is a solution of the EWOD Problem 3.4. Note that relation 42, can be rewritten

$$
b\left(\mathbf{u}_{\epsilon}, q\right)=0, \quad \forall q \in \mathbb{Q}
$$

and

$$
A_{\epsilon}\left(\left(\mathbf{u}_{\epsilon}, \lambda_{\epsilon}\right),\left(\mathbf{u}_{\epsilon}, \lambda_{\epsilon}\right)\right) \leqslant A_{\epsilon}\left(\left(\mathbf{u}_{\epsilon}, \lambda_{\epsilon}\right),(\mathbf{v}, \mu)\right)+b\left(\mathbf{v}, p_{\epsilon}\right)+\chi\left(\mathbf{u}_{\epsilon}-\mathbf{v}\right), \quad \forall(\mathbf{v}, \mu) \in \mathbb{V} \times \Lambda .
$$

Passing to the limit, we get $(\mathbf{w}, r, \xi) \in \mathcal{S}$ by invoking the lower semi-continuity property of the weak limit.

4.2.2 Uniqueness. The perturbed problem (42) will also be useful to prove the uniqueness of the solution. Indeed, it will be shown that the limiting problem has a unique solution characterized by relation (41). That is, the limit solution of the perturbed problem is the one with $\lambda$ of minimal norm.

Lemma 4.5 (Uniqueness) Assume $\mathcal{S} \neq \emptyset$ and for $\epsilon>0$ let $\left(\mathbf{u}_{\epsilon}, p_{\epsilon}, \lambda_{\epsilon}\right) \in \mathbb{V} \times \mathbb{Q} \times \mathbb{M}$ be the solution of 42). Then $\left(\mathbf{u}_{\epsilon}, p_{\epsilon}, \lambda_{\epsilon}\right) \rightarrow\left(\mathbf{u}_{0}, p_{0}, \lambda_{0}\right) \in \mathbb{V} \times \mathbb{Q} \times \mathbb{M}$, where $\left(\mathbf{u}_{0}, p_{0}, \lambda_{0}\right)$ is the solution in $\mathcal{S}$ with $\lambda_{0} \in \mathbb{M}$ of minimal $\mathbb{M}$ norm.

Proof. Again, we follow Theorem 3.2 in Lions-Stampacchia [41] but in a saddle point framework. Recalling that $\left(\mathbf{u}_{0}, p_{0}, \lambda_{0}\right) \in \mathcal{S}$ satisfies (40), we choose $(\mathbf{v}, q, \mu)=\left(\mathbf{u}_{\epsilon}, p_{\epsilon}, \lambda_{\epsilon}\right)$ in (40) and $(\mathbf{v}, q, \mu)=\left(\mathbf{u}_{0}, p_{0}, \lambda_{0}\right)$ in 42 , to obtain

$$
b\left(\mathbf{u}_{\epsilon}, p_{\epsilon}\right)=b\left(\mathbf{u}_{\epsilon}, p_{0}\right)=b\left(\mathbf{u}_{0}, p_{\epsilon}\right)=b\left(\mathbf{u}_{0}, p_{0}\right)=0 .
$$

In addition, the coercivity 39 of $a(\cdot, \cdot)$ yields

$$
c_{a}\left|\left\|\mathbf{u}_{\epsilon}-\mathbf{u}_{0} \mid\right\|_{\mathbb{V}}^{2}+\epsilon\left\langle\lambda_{\epsilon}-\lambda_{0}, \lambda_{\epsilon}\right\rangle_{\mathbb{M}} \leqslant 0,\right.
$$

whence $\mathbf{u}_{\epsilon} \rightarrow \mathbf{u}_{0}$ in $\mathbb{V}$. For the pinning, we know that $\left\|\lambda_{\epsilon}\right\|_{\mathbb{M}} \preceq P_{\text {pin }}$ by Lemma 3.2 The boundedness of the pressure is obtained via the inf-sup condition (45), similar to (48). Therefore, the existence of $(r, \xi) \in \mathbb{Q} \times \Lambda$ and a subsequence satisfying

$$
\left(p_{\eta}, \lambda_{\eta}\right) \rightarrow(r, \xi) \quad \text { in } \mathbb{Q} \times \Lambda
$$

is guaranteed. As in Lemma 4.4, we have $\left(\mathbf{u}_{0}, r, \xi\right) \in \mathcal{S}$.

It remains to show that $(r, \xi)=\left(p_{0}, \lambda_{0}\right)$ and that the weak convergence 50 is actually strong. The minimal norm property of $\lambda_{0}$ (recall (41)) implies that

$$
\left\langle\lambda_{0}, \xi-\lambda_{0}\right\rangle_{\mathbb{M}} \geqslant 0 .
$$


Moreover, relation (49) implies

$$
\left\langle\lambda_{0}-\lambda_{\epsilon}, \lambda_{\epsilon}\right\rangle_{\mathbb{M}} \geqslant 0,
$$

which becomes, by the semi-continuity property of the weak limit,

$$
\left\langle\lambda_{0}-\xi, \xi\right\rangle_{\mathbb{M}} \geqslant 0 \text {. }
$$

Adding (51) to (53) we get $\left\langle\lambda_{0}-\xi, \lambda_{0}-\xi\right\rangle_{\mathbb{M}} \leqslant 0$, i.e. $\xi=\lambda_{0}$ and $\lambda_{\epsilon} \rightarrow \lambda_{0}$ in $\mathbb{M}$. The strong convergence of $\lambda_{\epsilon}$ towards $\lambda_{0}$ is now obtained using (52):

$$
\left\|\lambda_{\epsilon}-\lambda_{0}\right\|_{\mathbb{M}}^{2}=\left\langle\lambda_{\epsilon}-\lambda_{0}, \lambda_{\epsilon}-\lambda_{0}\right\rangle_{\mathbb{M}}=\left\langle\lambda_{\epsilon}-\lambda_{0}, \lambda_{\epsilon}\right\rangle_{\mathbb{M}}+\left\langle\lambda_{0}-\lambda_{\epsilon}, \lambda_{0}\right\rangle_{\mathbb{M}} \leqslant\left\langle\lambda_{0}, \lambda_{0}-\lambda_{\epsilon}\right\rangle_{\mathbb{M}},
$$

so that the limit when $\epsilon \rightarrow 0$ implies

$$
\lambda_{\epsilon} \rightarrow \lambda_{0} \in \Lambda \text {. }
$$

Finally, we invoke the inf-sup (45) to recover the convergence of the pressure. Indeed, since $\left(\mathbf{u}_{0}, p_{0}, \lambda_{0}\right)$ and $\left(\mathbf{u}_{0}, r, \lambda_{0}\right)$ are both solutions of 40, , they belong to $\mathcal{S}$, hence

$$
b\left(\mathbf{v}, r-p_{0}\right)=0, \quad \forall \mathbf{v} \in \mathbb{V},
$$

and the inf-sup condition 45 leads to $r=p_{0}$ and $p_{\epsilon} \rightarrow p_{0}$. Similarly,

$$
b\left(\mathbf{v}, p_{0}-p_{\epsilon}\right)=a\left(\mathbf{u}_{\epsilon}-\mathbf{u}_{0}, \mathbf{v}\right)+c\left(\mathbf{v}, \lambda_{\epsilon}-\lambda_{0}\right) \leqslant\left(\left\|\mathbf{u}_{\epsilon}-\mathbf{u}_{0}\right\|_{\mathbb{V}}+\left\|\lambda_{\epsilon}-\lambda_{0}\right\|_{\mathbb{M}}\right)\||\mathbf{v}|\|_{\mathbb{V}},
$$

and again (45) implies $\left\|p_{0}-p_{\epsilon}\right\|_{\mathbb{Q}} \leqslant\left\|\mathbf{u}_{\epsilon}-\mathbf{u}_{0}\right\|_{\mathbb{V}}+\left\|\lambda_{\epsilon}-\lambda_{0}\right\|_{\mathbb{M}} \rightarrow 0$.

\subsection{Continuous inf-sup condition}

For completeness, we state and prove the inf-sup condition for pressures in $\mathbb{Q}$ with mean value zero and multipliers in $\mathbb{M}$. This will be useful for comparing the continuous and discrete solutions for error analysis (see Section 5.6.

LEMMA 4.6 (Continuous inf-sup) Let $\Omega$ be of class $W_{\infty}^{2}$. Then there exists a constant $\beta>0$ such that

$$
\sup _{\mathbf{v} \in \mathbb{V}} \frac{b(\mathbf{v}, q)+c(\mathbf{v}, \mu)}{\|\mathbf{v}\| \|_{\mathbb{V}}} \geqslant \beta\left(\|q\|_{\mathbb{Q}}^{2}+\|\mu\|_{\mathbb{M}}^{2}\right)^{1 / 2}
$$

for all $q$ in $\mathbb{Q}_{0}$, and $\mu$ in $\mathbb{M}$. Here, $\mathbb{Q}_{0}$ is the space of pressures with mean value zero.

Proof. Fix $q$ in $\mathbb{Q}_{0}$ and $\mu$ in $\mathbb{M}$. We will construct a velocity $\mathbf{v}$ that gives us (54). The proof is broken up into the following steps.

Step 1: Construct a vector function $\mathbf{v}_{0} \in H^{1}(\Gamma)$ such that

$$
\|\mu\|_{\mathbb{M}}=\left\langle\mu, \mathbf{v}_{0} \cdot \nu\right\rangle, \quad\left\|\mathbf{v}_{0}\right\|_{1, \Gamma} \leqslant c_{0}
$$

By the definition of the $\mathbb{M}$ norm $\left(26\right.$, there exists an $\eta_{0} \in \mathbb{G}$ such that $\left\|\eta_{0}\right\|_{\mathbb{G}}=1$ and $\|\mu\|_{\mathbb{M}}=$ $\left\langle\mu, \eta_{0}\right\rangle$. Next, define $\mathbf{v}_{0}=\eta_{0} \nu$. Because the domain is smooth, straightforward bounds show that $\left\|\mathbf{v}_{0}\right\|_{1, \Gamma} \leqslant c_{0}$ for some constant $c_{0}>0$. Ergo, $\mathbf{v}_{0}$ satisfies [55]. 
Step 2: Let $\mathbf{v} \in H^{1}(\Omega)$ satisfy the following divergence equation [28, 52]:

$$
\begin{aligned}
& \nabla \cdot \mathbf{v}=-q+\zeta \quad \text { in } \Omega \text {, } \\
& \mathbf{v}=\|\mu\|_{\mathbb{M}} \mathbf{v}_{0} \quad \text { on } \Gamma \text {, }
\end{aligned}
$$

where $\zeta$ is a constant to guarantee compatibility and is given by

$$
\zeta=\frac{\|\mu\|_{\mathbb{M}}}{|\Omega|} \int_{\Gamma} \mathbf{v}_{0} \cdot \nu
$$

Moreover, note the following inequality:

$$
|\zeta| \leqslant \frac{|\Gamma|^{1 / 2}}{|\Omega|}\|\mu\|_{\mathbb{M}}\left\|\mathbf{v}_{0}\right\|_{0, \Gamma} \leqslant c_{0} \frac{|\Gamma|^{1 / 2}}{|\Omega|}\|\mu\|_{\mathbb{M}}
$$

Therefore, using $\mathbf{v}_{0}$ from Step 1 , we find that the solution to 56 satisfies $\|\mathbf{v}\|_{1, \Gamma} \leqslant c_{0}\|\mu\|_{\mathbb{M}}$ and $\|\mathbf{v}\|_{H(\operatorname{div}, \Omega)} \leqslant c_{1}\left(\|q\|_{\mathbb{Q}}+\|\mathbf{v}\|_{1 / 2, \Gamma}\right) \leqslant c_{1}\left(\|q\|_{\mathbb{Q}}+\|\mu\|_{\mathbb{M}}\right)$. Hence, we get the bound

$$
\|\mathbf{v}\|_{\mathbb{V}} \leqslant c_{2}\left(\|q\|_{\mathbb{Q}}+\|\mu\|_{\mathbb{M}}\right) .
$$

Note that $\mathbf{v}$ is contained in $\mathbb{V}$ by 20 .

Step 3: Insert the function $\mathbf{v}$ from Step 2 into $b(\cdot, q)+c(\cdot, \mu)$ :

$$
\frac{b(\mathbf{v}, q)+c(\mathbf{v}, \mu)}{\|\mathbf{v}\|_{\mathbb{V}}}=\frac{-\int_{\Omega} q \nabla \cdot \mathbf{v}+\langle\mu, \mathbf{v} \cdot \boldsymbol{\nu}\rangle}{\|\mathbf{v}\|_{\mathbb{V}}}=\frac{\int_{\Omega} q^{2}-\zeta \int_{\Omega} q+\|\mu\|_{\mathbb{M}}\left\langle\mu, \mathbf{v}_{0} \cdot \boldsymbol{\nu}\right\rangle}{\|\mathbf{v}\|_{\mathbb{V}}}
$$

By Step 1, and since $q$ has mean value zero, we get

$$
\frac{b(\mathbf{v}, q)+c(\mathbf{v}, \mu)}{\|\mathbf{v}\|_{\mathbb{V}}}=\frac{\|q\|_{\mathbb{Q}}^{2}+\|\mu\|_{\mathbb{M}}^{2}}{\|\mathbf{v}\|_{\mathbb{V}}} .
$$

Step 4: Use the bound given in 57) and take the supremum over all of $\mathbb{V}$ to get

$$
\sup _{\mathbf{v} \in \mathbb{V}} \frac{b(\mathbf{v}, q)+c(\mathbf{v}, \mu)}{\|\mathbf{v}\| \mathbb{V}} \geqslant \beta\left(\|q\|_{\mathbb{Q}}^{2}+\|\mu\|_{\mathbb{M}}^{2}\right)^{1 / 2}
$$

for some fixed constant $\beta>0$. Since $q$ and $\mu$ were arbitrary, this proves the assertion.

REMARK 4.7 (Inf-sup for pressure with mean value zero) The proof given here follows a standard argument and can be found in [53], where the function spaces were slightly different. Note the infsup property (54) only holds for pressures with zero mean value, so it is consistent with the lack of uniqueness discussed in Section 4.1. In view of this, it is clear that the perturbation argument in Section 4.2 selects the constant part of $\lambda$ such that it minimizes its $\mathbb{M}$ norm. Also note that full uniqueness is guaranteed if $\lambda=P_{\text {pin }}$ and $\lambda=-P_{\text {pin }}$ on disjoint parts of the boundary (see Lemma 6.1. 


\subsection{Expected regularity}

The regularity theory for the time-discrete problem is open. We discuss here the most plausible regularity of the triple $(\mathbf{u}, p, \lambda)$ and later assume it in the error analysis of Subsection 5.6

We start by recalling Lemma 3.2 , namely

$$
\Lambda \subset L^{\infty}(\Gamma)
$$

The definition of the convex set 27] is thus equivalent to the following alternative form:

$$
\Lambda=\left\{\mu \in L^{\infty}(\Gamma):|\mu| \leqslant P_{\text {pin }}\right\}
$$

We assume that the boundary $\Gamma$ is at least of class $W_{\infty}^{2}$, so that curvature makes sense, and that there exists at least one point $\mathbf{x}$ on $\Gamma$ across which the normal velocity $\mathbf{u} \cdot \boldsymbol{\nu}$ changes sign. Because of (2), this translates into:

The multiplier $\lambda$ has a jump discontinuity at $\mathrm{x} \in \Gamma$.

This seems to be a typical situation according to the simulation of Section 3.6 (see Figure 2). Thus, the following argument indicates that the boundary regularity of $(\mathbf{u}, p, \lambda)$ is limited to

$$
\mathbf{u} \in W_{\infty}^{2}(\Gamma), \quad p \in H^{1 / 2}(\Gamma), \quad \lambda \in B V(\Gamma) \backslash H^{1 / 2}(\Gamma),
$$

i.e., $\lambda$ is a bounded variation function on $\Gamma$ which is not in $H^{1 / 2}$. First note that the regularity (62) of $\lambda$ is compatible with (61). Secondly, choosing a test function $\mathbf{v}$ in $\mathbb{V}$ in 40] with compact support yields $-\nabla p=\alpha \frac{\mathbf{u}-\mathbf{u}^{\text {old }}}{\delta t}+\beta \mathbf{u} \in L^{2}(\Omega)$, whence $p \in H^{1 / 2}(\Gamma)$. Now consider the boundary condition (11) (or (12)) with EWOD forcing $E \in H^{1 / 2}(\Gamma)$. Since $\mathbf{u} \cdot \nu \in H^{1}(\Gamma)$ by construction, we have

$$
p \boldsymbol{\nu}+\Delta_{\Gamma} \mathbf{x}+\delta t \Delta_{\Gamma} \mathbf{u} \in L^{\infty}(\Gamma) \backslash H^{1 / 2}(\Gamma),
$$

In view of the relation (8) between $\mathbf{x}, \mathbf{u}$ and $\mathbf{X}$ to update the interface $\Gamma$, we expect $\mathbf{x}$ and $\mathbf{u}$ to have the same regularity, which is also consistent with $(63)$. Consequently, the maximal boundary regularity of $\mathbf{x}, \mathbf{u}$ compatible with $(63)$ is $\mathbf{x}, \mathbf{u} \in W_{\infty}^{2}(\Gamma) \backslash H^{5 / 2}(\Gamma)$.

Since $\mathrm{x}$ is the identity on $\Gamma$, the vector curvature $\kappa \nu=-\Delta_{\Gamma} \mathrm{x}$ of $\Gamma$ is just bounded for $\Gamma \in$ $W_{\infty}^{2}$. This plausibility argument reveals that a jump of $\lambda \in P_{\operatorname{pin}} \operatorname{sgn}(\mathbf{u} \cdot \boldsymbol{\nu})$ is compensated by a corresponding curvature jump, which is at most in $L^{\infty}(\Gamma) \cap B V(\Gamma)$.

\section{Finite element discretization}

The stability of the finite element scheme requires the velocity space to be rich enough to accommodate the pressure $p_{h}$ and the boundary multiplier $\lambda_{h}$ [10]. In Lemma 5.4. we show that continuous piecewise quadratic polynomial velocities satisfy this property for $p_{h}$ and $\lambda_{h}$ continuous piecewise linear. To maintain accuracy and avoid numerical artifacts due to polynomial mismatch [7. 9] (see Remark 5.2), the shape of the domain must be properly approximated. We resort to the use of iso-parametric elements (see for instance [9]), and state the fully discrete version of Problem 3.4. We end this section by describing the interpolation operators needed in the convergence study of the finite element scheme; see Corollary 5.7 and Remark 5.10 


\subsection{Iso-parametric finite elements}

We start by approximating the boundary $\Gamma$ by a (continuous) piecewise quadratic boundary. Given the quasi-uniform mesh size $h>0$, let $\left\{x_{i}\right\}_{i=1}^{J}$ be a set of points lying on $\Gamma$ such that the distance between two consecutive points does not exceed $h$. Define the discrete approximation of $\Gamma$ by

$$
\Gamma_{h}:=\left\{\mathbf{X}_{h}(s): s \in I\right\}
$$

where $\mathbf{X}_{h}$ is the continuous, piecewise quadratic interpolant of $\mathbf{X}$ related to the partition $\left\{x_{i}\right\}_{i=1}^{J}$ of $\Gamma$ (compare to 9 ). Then we partition the enclosed domain $\Omega_{h}$ consisting of a set of triangles with diameters $h$. Denote this set of triangles by $\mathrm{T}_{\Omega_{h}}$ and the set of sides of $\Gamma_{h}$ by $\mathrm{S}_{\Gamma_{h}}$. Note that each side in $\mathrm{S}_{\Gamma_{h}}$ is a quadratic curve, with normal vector $\nu_{h}$. Ergo, any triangle in $\mathrm{T}_{\Omega_{h}}$ that lies on the boundary will be curved. The choice of quadratics is dictated by stability, and that of iso-parametric quadratics to maintain accuracy [9, Section 10.4] and geometric consistency [7].

Next, we introduce the polynomial spaces that will be used in approximating $\mathbb{V}, \mathbb{Q}$, and $\mathbb{M}$. Let $\mathcal{P}_{k}$ be the space of polynomials of degree $\leqslant k$ on the standard reference triangle $\widehat{T}$ or standard reference side $\widehat{S}$. Let $\Psi_{T}: \widehat{T} \rightarrow T$ be the iso-parametric $\mathcal{P}_{2}$ mapping from the reference triangle to a triangle in $\mathrm{T}_{\Omega_{h}}$, and let $\Psi_{S}: \widehat{S} \rightarrow S$ be similarly defined for boundary sides $S$. Then the finite element spaces are defined as follows. Let

$$
V_{k}:=\left\{\mathbf{v} \in\left[C\left(\bar{\Omega}_{h}\right)\right]^{2}: \mathbf{v} \circ \Psi_{T} \in \mathcal{P}_{k}(\widehat{T}), \forall T \in \mathrm{T}_{\Omega_{h}}\right\}
$$

be a space of continuous vector basis functions, which are piecewise polynomials of degree $\leqslant k$ on the reference triangle $\widehat{T}$, and

$$
Q_{k}:=\left\{q \in C\left(\bar{\Omega}_{h}\right): q \circ \Psi_{T} \in \mathcal{P}_{k}(\widehat{T}), \forall T \in \mathrm{T}_{\Omega_{h}}\right\}
$$

be a space of continuous scalar basis functions of degree $\leqslant k$; note that $V_{k} \subset\left[H^{1}\left(\Omega_{h}\right)\right]^{2}, Q_{k} \subset$ $L^{2}\left(\Omega_{h}\right)$. We also have a continuous finite element space $M_{k} \subset L^{2}\left(\Gamma_{h}\right)$,

$$
M_{k}:=\left\{\mu \in C\left(\Gamma_{h}\right): \mu \circ \Psi_{S} \in \mathcal{P}_{k}(\widehat{S}), \forall S \in \mathrm{S}_{\Gamma_{h}}\right\}
$$

for $k \geqslant 1$, or a space of piecewise constant elements $\mathcal{P}_{0}$ for $k=0$.

Let $\mathbb{V}_{h}, \mathbb{Q}_{h}$, and $\mathbb{M}_{h}$ be approximations of $\mathbb{V}, \mathbb{Q}$, and $\mathbb{M}$ respectively defined by

$$
\mathbb{V}_{h}:=V_{2}, \quad \mathbb{Q}_{h}:=Q_{1}, \quad \mathbb{M}_{h}:=M_{1},
$$

or $\mathbb{M}_{h}:=M_{0}$. These finite element spaces are equipped with the norms $\|\cdot\|\left\|_{\mathbb{V}_{h}},\right\| \cdot\left\|_{\mathbb{Q}_{h}},\right\| \cdot \|_{\mathbb{M}_{h}}$, which are the discrete counterparts to the continuous ones (22), (24) and (26).

Finally, set $\Lambda_{h}:=\left\{\mu_{h} \in \mathbb{M}_{h}:\left|\mu_{h}\right| \leqslant P_{\text {pin }}\right\}$ as an approximation of the convex set and define $\mathbb{G}_{h}$ as an approximation of $\mathbb{G}($ recall $[25)$ by

$$
\mathbb{G}_{h}:=\left\{\eta_{h}: \eta_{h}=\mathbf{v}_{h} \cdot \boldsymbol{\nu}_{h}, \text { where } \mathbf{v}_{h} \in \mathbb{V}_{h}\right\} .
$$

Note that the variational crime argument in Section 5.4 implies $\mathbb{G}_{h} \subset \mathbb{G}$ (see (75)).

REMARK 5.1 (Velocity space) The pair $\mathbb{V}_{h}, \mathbb{Q}_{h}$ is exactly the same as the popular $\mathcal{P}_{2}-\mathcal{P}_{1}$ TaylorHood element used for the Stokes equations [10], [29]. It is not obvious that a lower degree space for the velocity, such as the lowest order MINI element [2], would ensure that the inf-sup condition is satisfied for the triple $\left(\mathbb{V}_{h}, \mathbb{Q}_{h}, \mathbb{M}_{h}\right)$. A velocity space with extra degrees of freedom on $\Gamma$ is required to account for the extra Lagrange multiplier $\lambda$ (cf. [53]). 
REMARK 5.2 (Polynomial mismatch) We stress that the combination of quadratic iso-parametric boundary edges with Taylor-Hood finite elements is essential for accurate computation of curvature. Recall that the boundary position is updated via a piecewise quadratic velocity according to [8]-(9). Using straight instead of iso-parametric boundary edges would give a polynomial mismatch resulting in a geometric inconsistency. This manifests as a suboptimal rate of convergence for curvature, in fact worse than using straight edges and piecewise linear velocity. This is examined in [7. Section 4.4].

\subsection{The fully discrete EWOD problem with pinning}

First, we define the discrete counterpart of the forms $a(\cdot, \cdot), b(\cdot, \cdot), c(\cdot, \cdot)$ and $\chi(\cdot)$ (see (36)-(38)). Recall that we define $\mathbf{u}^{\text {old }}:=\mathbf{u}^{i}$ to be the velocity at the previous time step and we drop the time index ' $i$ ' notation: Given approximations $E_{h} \in \mathbb{V}_{h}^{*}$ and $\mathbf{u}_{h}^{\text {old }} \in \mathbb{V}_{h}$ of the electrowetting force $E \in \mathbb{V}^{*}$ and previous velocity $\mathbf{u}^{\text {old }} \in \mathbb{V}$, we define

$$
\begin{aligned}
a_{h}\left(\mathbf{u}_{h}, \mathbf{v}_{h}\right)= & \left(\frac{\alpha}{\delta t}+\beta\right)\left(\int_{\Omega_{h}} \mathbf{u}_{h} \cdot \mathbf{v}_{h}+\gamma \int_{\Omega_{h}}\left(\nabla \cdot \mathbf{u}_{h}\right)\left(\nabla \cdot \mathbf{v}_{h}\right)\right) \\
& +D_{\mathrm{visc}} \int_{\Gamma_{h}}\left(\mathbf{u}_{h} \cdot \nu_{h}\right)\left(\mathbf{v}_{h} \cdot \boldsymbol{\nu}_{h}\right)+\delta t \int_{\Gamma_{h}} \nabla_{\Gamma_{h}} \mathbf{u}_{h}: \nabla_{\Gamma_{h}} \mathbf{v}_{h}, \\
b_{h}\left(\mathbf{v}_{h}, q_{h}\right)= & -\int_{\Omega_{h}} q_{h} \nabla \cdot \mathbf{v}_{h}, \quad c_{h}\left(\mathbf{v}_{h}, \mu_{h}\right)=\int_{\Gamma_{h}} \mu_{h} \mathbf{v}_{h} \cdot \nu_{h}, \\
\chi\left(\mathbf{v}_{h}\right)= & \frac{\alpha}{\delta t} \int_{\Omega_{h}} \mathbf{u}_{h}^{\text {old }} \cdot \mathbf{v}_{h}-\int_{\Gamma_{h}} E_{h} \mathbf{v}_{h} \cdot \nu_{h}-\int_{\Gamma_{h}} \nabla_{\Gamma_{h}} \mathbf{x}: \nabla_{\Gamma_{h}} \mathbf{v}_{h},
\end{aligned}
$$

for all $\mathbf{v}_{h} \in \mathbb{V}_{h}, q_{h} \in \mathbb{Q}_{h}$ and $\mu_{h} \in \mathbb{M}_{h}$. The variational formulation of the space-discrete version of Problem 3.4 then reads as follows.

PRoBlem 5.3 (Fully discrete EWOD with pinning) Let $\chi \in \mathbb{V}_{h}^{*}$ be given data. Then we say that $\mathbf{u}_{h} \in \mathbb{V}_{h}, p_{h} \in \mathbb{Q}_{h}$, and $\lambda_{h} \in \Lambda_{h}$ is a solution of the nonlinear problem if

$$
\begin{aligned}
& a_{h}\left(\mathbf{u}_{h}, \mathbf{v}_{h}\right)+b_{h}\left(\mathbf{v}_{h}, p_{h}\right)+c_{h}\left(\mathbf{v}_{h}, \lambda_{h}\right)=\chi\left(\mathbf{v}_{h}\right), \\
& b_{h}\left(\mathbf{u}_{h}, q_{h}\right)=0, \quad c_{h}\left(\mathbf{u}_{h}, \mu_{h}-\lambda_{h}\right) \leqslant 0,
\end{aligned}
$$

for all $\mathbf{v}_{h} \in \mathbb{V}_{h}, q_{h} \in \mathbb{Q}_{h}$ and $\mu_{h} \in \Lambda_{h}$.

As in the continuous case, the well-posedness and in particular the lack of uniqueness discussed in Section 4.1 can be handled by adding a coercive term $\epsilon\left\langle\lambda_{h}, \mu_{h}-\lambda_{h}\right\rangle_{\mathbb{M}_{h}}$ to the variational inequality. This selects from all possible solutions $\lambda_{h}$ one with minimal $\mathbb{M}$ norm. However, adding a coercive term, with finite $\epsilon$ in practice, would alter the contact line pinning term $P_{\text {pin }} \operatorname{sgn}(\mathbf{u} \cdot \boldsymbol{\nu})$ and would not correctly capture the physics of liquid-gas interface motion with pinning.

With this in mind, we note that uniqueness for the semi-discrete Problem 3.4 can be shown via Lemma4.6 (continuous inf-sup condition) with the caveat that $p$ and $\lambda$ may be shifted by a constant such that $p-\lambda$ remains fixed. Thus, we will prove the convergence of the finite element solution toward the solution of the EWOD Problem 3.4 with an appropriate constant shift of $p_{h}$ and $\lambda_{h}$. In practice, this does not affect the velocity and therefore the droplet evolution (see Sections 6, 77and Remark 6.2. 


\subsection{The interpolation operators}

This section discusses the interpolation operator onto $\mathbb{V}_{h} \times \mathbb{Q}_{h} \times \Lambda_{h}$. The choice adopted here for the velocity and pressure space is classical: let $\pi_{\mathbb{V}_{h}}:\left[L^{1}\left(\Omega_{h}\right)\right]^{2} \rightarrow \mathbb{V}_{h}$ and $\pi_{\mathbb{Q}_{h}}: L^{1}\left(\Omega_{h}\right) \rightarrow \mathbb{Q}_{h}$ denote the Clément interpolation operators [16]. Regarding the boundary multiplier space $\mathbb{M}_{h}$, we define a Clément interpolation operator $\pi_{\Lambda_{h}}$ as follows. Let $\left\{\mathbf{x}_{j}\right\}_{j=1}^{J}$ be the boundary vertices of $\Gamma_{h}$ and $\left\{\omega_{j}\right\}_{j=1}^{J}$ be the boundary stars, i.e. $\omega_{j}=S \cup S^{\prime}$ with $S, S^{\prime} \in \mathrm{S}_{\Gamma_{h}}$ and $\mathrm{x}_{j}=\bar{S} \cap \overline{S^{\prime}}$. Then

$$
\pi_{\Lambda_{h}} \mu(\mathbf{x}):=\sum_{j=1}^{J}\left(\frac{1}{\left|\omega_{j}\right|} \int_{\omega_{j}} \mu\right) \phi_{j}(\mathbf{x})
$$

where $\phi_{j}$ is a piecewise linear 'hat' function centered at $\mathbf{x}$. This operator has the following fundamental properties:

- $\pi_{\Lambda_{h}}$ is stable in $L^{1}(\Gamma)$;

- $\pi_{\Lambda_{h}}$ preserves the bound $|\mu| \leqslant P_{\text {pin }}:\left|\pi_{\Lambda_{h}} \mu\right| \leqslant P_{\text {pin }}$;

- $\pi_{\Lambda_{h}}$ has optimal approximation properties in $L^{1}(\Gamma)$ :

$$
\left\|\mu-\pi_{\Lambda_{h}} \mu\right\|_{L^{1}(S)} \lesssim h\|\mu\|_{B V\left(\omega_{S}\right)}, \quad \forall S \in \mathrm{S}_{\Gamma_{h}}
$$

where $B V\left(\omega_{S}\right)$ is the space of functions of bounded variation over the star $\omega_{S}$. This can be shown by regularization in $W_{1}^{1}\left(\Gamma_{h}\right)$ and interpolation in $W_{1}^{1}\left(\Gamma_{h}\right)$ [9].

\subsection{Variational crime}

The solution $(\mathbf{u}, p, \lambda)$ of the semi-discrete Problem 3.4 is defined on the smooth domain $\Omega$ whereas the solution $\left(\mathbf{u}_{h}, p_{h}, \lambda_{h}\right)$ of the fully discrete Problem 5.3 is defined on a domain $\Omega_{h}$ with piecewise quadratic, globally Lipschitz boundary. Therefore, the error in the finite element approximation contains two terms: the energy error between the semi-discrete and fully discrete solutions and the variational crime related to the domain approximation. The latter is of order $O\left(h^{2}\right)$, according to [38], [9, Section 10.4], and is better than the expected $O(h)$ error due to the variational inequality (see Corollary 5.7 below). Therefore, to avoid technicalities we hereafter assume, except in Step 1 of Lemma 5.5 that the semi-discrete Problem 3.4 is defined over the polygonal domain $\Omega_{h}$ but that the regularity of its solution $(\mathbf{u}, p, \lambda)$ is unaffected. We thus assume $\Omega_{h} \equiv \Omega, \Gamma_{h} \equiv \Gamma$ and, recalling [20], that

$$
\mathbb{V}_{h} \subset \mathbb{V}, \quad \mathbb{Q}_{h} \subset \mathbb{Q}, \quad \mathbb{M}_{h} \subset \mathbb{M}, \quad \Lambda_{h} \subset \Lambda, \quad \mathbb{G}_{h} \subset \mathbb{G} .
$$

Note this also implies $\left\|\left|\cdot\left\|\left.\right|_{\mathbb{V}_{h}} \equiv\right\|\|\cdot\|\left\|_{\mathbb{V}},\right\| \cdot\left\|_{\mathbb{Q}_{h}} \equiv\right\| \cdot\left\|_{\mathbb{Q}},\right\| \cdot\left\|_{\mathbb{G}_{h}} \equiv\right\| \cdot \|_{\mathbb{G}}\right.\right.$, and $\|\cdot\|_{\mathbb{M}_{h}} \equiv\|\cdot\|_{\mathbb{M}}$.

\subsection{Inf-sup condition}

The convergence of the solution of Problem 5.3 towards the solution of Problem 3.4 relies on the following inf-sup condition. 
Lemma 5.4 (Discrete inf-sup) Let $\Omega$ be of class $W_{\infty}^{2}$. Then there exist constants $\beta>0$ and $h_{1}>0$, which do not depend on $h$, such that

$$
\sup _{\mathbf{v}_{h} \in \mathbb{V}_{h}} \frac{b\left(\mathbf{v}_{h}, q_{h}\right)+c\left(\mathbf{v}_{h}, \mu_{h}\right)}{\left\|\mathbf{v}_{h}\right\| \| \mathbb{V}} \geqslant \beta\left(\left\|q_{h}\right\|_{\mathbb{Q}}^{2}+\left\|\mu_{h}\right\|_{\mathbb{M}}^{2}\right)^{1 / 2}
$$

for all $q_{h}$ in $\mathbb{Q}_{h, 0}, \mu_{h}$ in $\mathbb{M}_{h}$ and $h \leqslant h_{1}$. This is the discrete analog of Lemma 4.6.

The proof of Lemma 5.4 is split into two stages. First, we prove it for the pair $\left(\mathbb{V}_{h}, \mathbb{M}_{h}\right)$, and then we combine this with the known result for the Taylor-Hood $\left(\mathbb{V}_{h}, \mathbb{Q}_{h}\right)$ inf-sup condition to show the entire inf-sup.

LEMMA 5.5 (Inf-sup for the boundary multiplier) Let $\Omega$ be of class $W_{\infty}^{2}$. Then there are constants $\beta_{2}>0$ and $h_{1}>0$, which do not depend on $h$, such that

$$
\sup _{\mathbf{v}_{h} \in \mathbb{V}_{h}} \frac{c\left(\mathbf{v}_{h}, \mu_{h}\right)}{\left\|\mathbf{v}_{h}\right\|_{\mathbb{V}}} \geqslant \beta_{2}\left\|\mu_{h}\right\|_{\mathbb{M}} \quad \text { for all } \mu_{h} \in \mathbb{M}_{h} \text { and all } h \leqslant h_{1} .
$$

Proof. Step 1: The main contribution here is the construction of a special vector function $\boldsymbol{\nu}_{s}$ on $\Gamma_{h}$ that has the following properties:

$$
\boldsymbol{\nu}_{s} \in M_{1}, \quad\left\|\boldsymbol{\nu}_{s}\right\|_{1, \Gamma_{h}} \leqslant a_{1}, \quad \boldsymbol{\nu}_{s} \cdot \boldsymbol{\nu}_{h} \geqslant a_{2}>0,
$$

for some constants $a_{1}, a_{2}$ independent of $h$. We recall that the piecewise quadratic polygonal boundary $\Gamma_{h}$ approximates a $W_{\infty}^{2}$ boundary $\Gamma$ with $W_{\infty}^{1}$ unit normal vector $\boldsymbol{\nu}$. With this in mind, we let $F: \Gamma_{h} \rightarrow \Gamma$ be a Lipschitz, piecewise smooth map from the discrete boundary to the smooth boundary. Assuming $h$ is sufficiently small, this map can be built locally on each side $S \in \mathrm{S}_{\Gamma_{h}}$ [1]. In addition, for $h$ small enough, $\boldsymbol{\nu}_{h} \cdot(\boldsymbol{\nu} \circ F) \geqslant 2 / 3$ on all of $\Gamma_{h}$. Since $\boldsymbol{\nu}$ is smooth and bounded, and $F$ is piecewise smooth, $\|\boldsymbol{\nu} \circ F\|_{W_{\infty}^{1}\left(\Gamma_{h}\right)} \leqslant c$, with $c$ independent of $h$ but dependent on the curvature of $\Gamma$.

Now let $\boldsymbol{\nu}_{s} \in M_{1}$ be a continuous piecewise finite element function on $\Gamma_{h}$ such that $\boldsymbol{\nu}_{s}\left(\mathrm{x}_{i}\right):=$ $(\boldsymbol{\nu} \circ F)\left(\mathbf{x}_{i}\right)$. This implies that $\boldsymbol{\nu}_{s} \in\left[W_{\infty}^{1}\left(\Gamma_{h}\right)\right]^{2}$ by interpolation theory [9], and $\boldsymbol{\nu}_{h} \cdot \boldsymbol{\nu}_{s}\left(\mathbf{x}_{i}\right) \geqslant 2 / 3$ at each vertex $\mathrm{x}_{i}$. Since the sides of $\Gamma_{h}$ are quadratic, it is straightforward to show that $\left.\nu_{h}\right|_{S}=$ $\nu_{c}+O(h)$ on each side $S$, where $\nu_{c}$ is a constant vector (because $\Gamma$ is smooth). So, for $h$ sufficiently small, $\boldsymbol{\nu}_{h} \cdot \boldsymbol{\nu}_{s} \geqslant 1 / 2$, whence $\boldsymbol{\nu}_{s}$ satisfies (78).

Step 2: $\quad$ Let $\mu_{h} \in \mathbb{M}_{h}$ and define $\mathbf{u}_{h} \in \mathbb{V}_{h}$ by $\left.\mathbf{u}_{h}\right|_{\Gamma}:=\mu_{h} \boldsymbol{\nu}_{s}$ (note: both $\mu_{h}$ and $\boldsymbol{\nu}_{s}$ are linear on the reference element, so their product is a quadratic function). Next, extend $\mathbf{u}_{h}$ in the following way. Let $\mathbf{w} \in\left[H^{1}\left(\Omega_{h}\right)\right]^{2} \cap\left[H^{1}\left(\Gamma_{h}\right)\right]^{2}$ satisfy the following divergence equation (see [54, 8 , 22, [29]):

$$
\begin{aligned}
\nabla \cdot \mathbf{w} & =\frac{1}{\left|\Omega_{h}\right|} \int_{\Gamma_{h}} \mathbf{u}_{h} \cdot \boldsymbol{\nu}_{h}=\frac{1}{\left|\Omega_{h}\right|} \int_{\Gamma_{h}} \mu_{h}\left(\boldsymbol{\nu}_{s} \cdot \boldsymbol{\nu}_{h}\right) \quad \text { in } \Omega_{h}, \\
\mathbf{w} & =\mathbf{u}_{h}=\mu_{h} \boldsymbol{\nu}_{s} \quad \text { on } \Gamma_{h} .
\end{aligned}
$$

By the properties (78) of $\boldsymbol{\nu}_{s}$ and an inverse estimate $\left\|\mathbf{u}_{h}\right\|_{1 / 2, \Gamma_{h}} \leqslant C h^{-1 / 2}\left\|\mathbf{u}_{h}\right\|_{0, \Gamma_{h}}$, we have

$$
\|\mathbf{w}\|_{1, \Omega_{h}} \leqslant c_{0} h^{-1 / 2}\left\|\mu_{h}\right\|_{0, \Gamma_{h}},\|\mathbf{w}\|_{1, \Gamma_{h}} \leqslant c_{1} h^{-1}\left\|\mu_{h}\right\|_{0, \Gamma_{h}}, \quad \text { so } \quad\|\mathbf{w}\|_{\mathbb{V}} \leqslant c_{2} h^{-1}\left\|\mu_{h}\right\|_{0, \Gamma_{h}} .
$$


Next, let $\Pi_{h}$ be the Scott-Zhang interpolant [49] (onto $\mathbb{V}_{h}$ ), with the following modification. If the function to be interpolated is continuous, piecewise quadratic on $\Gamma_{h}$, then use standard interpolation for the boundary values of the interpolant. Therefore, setting $\mathbf{w}_{h}:=\Pi_{h} \mathbf{w}$, we have $\left.\mathbf{w}_{h}\right|_{\Gamma_{h}}=$ $\left.\mathbf{w}\right|_{\Gamma_{h}}=\mu_{h} \boldsymbol{\nu}_{s}$ and $\left\|\mathbf{w}_{h}\right\|\left\|_{\mathbb{V}} \leqslant c_{3} h^{-1}\right\| \mu_{h} \|_{0, \Gamma_{h}}$. This gives the following relation which indicates an inf-sup condition with a constant that depends on $h$ :

$$
\frac{c\left(\mathbf{w}_{h}, \mu_{h}\right)}{\left\|\mathbf{w}_{h}\right\| \|_{\mathbb{V}}} \geqslant c_{4} h \frac{\int_{\Gamma} \mu_{h}^{2}}{\left\|\mu_{h}\right\|_{0, \Gamma}}=c_{4} h\left\|\mu_{h}\right\|_{0, \Gamma} .
$$

Step 3: We need another vector function to account for the $h$ weighting. By the definition of the $\mathbb{M}$ norm (26), there exists a $\hat{\mathbf{v}} \in\left[H^{1}\left(\Gamma_{h}\right)\right]^{2}$ such that

$$
c\left(\hat{\mathbf{v}}, \mu_{h}\right)=\left\|\mu_{h}\right\|_{\mathbb{M}}, \quad\|\hat{\mathbf{v}}\|_{1, \Gamma_{h}}=1 .
$$

Next, extend $\hat{\mathbf{v}}$ to all of $\Omega_{h}$ just as was done in Step 2. Clearly, we have the bound $\|\hat{\mathbf{v}}\|_{1, \Omega}+$ $\|\hat{\mathbf{v}}\|_{1, \Gamma} \leqslant c_{5}\|\hat{\mathbf{v}}\|_{1, \Gamma}=c_{5}$. Let $\hat{\mathbf{v}}_{h}:=\Pi_{h} \hat{\mathbf{v}} \in \mathbb{V}_{h}$ be the Scott-Zhang interpolant onto $\mathbb{V}_{h}$, whence $\left\|\hat{\mathbf{v}}_{h}-\hat{\mathbf{v}}\right\|_{0, \Gamma} \leqslant c_{6} h\|\hat{\mathbf{v}}\|_{1, \Gamma}=c_{6} h$. Next, we have

$$
\begin{aligned}
c\left(\hat{\mathbf{v}}_{h}, \mu_{h}\right) & =c\left(\hat{\mathbf{v}}, \mu_{h}\right)+c\left(\hat{\mathbf{v}}_{h}-\hat{\mathbf{v}}, \mu_{h}\right) \\
& \geqslant\left\|\mu_{h}\right\|_{\mathbb{M}}-\left\|\hat{\mathbf{v}}_{h}-\hat{\mathbf{v}}\right\|_{0, \Gamma}\left\|\mu_{h}\right\|_{0, \Gamma} \geqslant\left\|\mu_{h}\right\|_{\mathbb{M}}-c_{6} h\left\|\mu_{h}\right\|_{0, \Gamma},
\end{aligned}
$$

where we used 81 and the Cauchy-Schwarz inequality.

Step 4: Combine the two discrete velocity vectors. Let $\mathbf{z}_{h}=\delta \mathbf{w}_{h} /\|\| \mathbf{w}_{h}\|\| \mathbb{V}+\hat{\mathbf{v}}_{h}$, where $\delta>0$ is a constant to be chosen. Then by [80] and [82] we get

$$
c\left(\mathbf{z}_{h}, \mu_{h}\right)=\delta \frac{c\left(\mathbf{w}_{h}, \mu_{h}\right)}{\left\|\mathbf{w}_{h}\right\| \|_{\mathbb{V}}}+c\left(\hat{\mathbf{v}}_{h}, \mu_{h}\right) \geqslant\left\|\mu_{h}\right\|_{\mathbb{M}}+h\left\|\mu_{h}\right\|_{0, \Gamma_{h}}\left(\delta c_{4}-c_{6}\right)=\left\|\mu_{h}\right\|_{\mathbb{M}}
$$

for $\delta:=c_{6} / c_{4}$. And we have the bound $\left\|\mid \mathbf{z}_{h}\right\|_{\mathbb{V}} \leqslant \delta+\left\|\hat{\mathbf{v}}_{h}\right\|_{\mathbb{V}} \leqslant c_{6} / c_{4}+c_{7}$. Therefore,

$$
\sup _{\mathbf{v}_{h} \in \mathbb{V}_{h}} \frac{c\left(\mathbf{v}_{h}, \mu_{h}\right)}{\left\|\mathbf{v}_{h} \mid\right\| \mathbb{V}} \geqslant \beta_{2}\left\|\mu_{h}\right\|_{\mathbb{M}}, \quad \beta_{2}=\frac{1}{c_{6} / c_{4}+c_{7}},
$$

which is the partial inf-sup condition for arbitrary $\mu_{h} \in \mathbb{M}_{h}$.

Proof of Lemma 5.4. Let $q_{h} \in \mathbb{Q}_{h, 0}$ and $\mu_{h} \in \mathbb{M}_{h}$ be arbitrary.

Step 1: By Lemma 5.5, there exists an $h_{1}>0$ and $\hat{\mathbf{u}}_{h} \in \mathbb{V}_{h}$ such that

$$
\int_{\Gamma_{h}} \mu_{h}\left(\hat{\mathbf{u}}_{h} \cdot \boldsymbol{\nu}\right) \geqslant \beta_{2}\left\|\mu_{h}\right\|_{\mathbb{M}}, \quad\left\|\hat{\mathbf{u}}_{h}\right\|_{\mathbb{V}}=1,
$$

for all $h \leqslant h_{1}$. Next, consider the following discrete Stokes problem, which has a unique solution $\left(\mathbf{u}_{h}^{*}, p_{h}^{*}\right) \in \mathbb{V}_{h, 0} \times \mathbb{Q}_{h, 0}$ [10], [8]:

$$
\begin{aligned}
\int_{\Omega_{h}} \nabla \mathbf{u}_{h}^{*}: \nabla \mathbf{v}_{h} & -\int_{\Omega_{h}} p_{h}^{*} \nabla \cdot \mathbf{v}_{h}=0 \\
& -\int_{\Omega_{h}} \rho_{h} \nabla \cdot \mathbf{u}_{h}^{*}=\int_{\Omega_{h}} \rho_{h}\left(\frac{q_{h}}{\left\|q_{h}\right\|_{\mathbb{Q}}}+\nabla \cdot \hat{\mathbf{u}}_{h}\right),
\end{aligned}
$$

for all $\mathbf{v}_{h} \in \mathbb{V}_{h, 0}$ and $\rho_{h} \in \mathbb{Q}_{h, 0}$, where $\mathbb{V}_{h, 0}=\mathbb{V}_{h} \cap H_{0}^{1}\left(\Omega_{h}\right)$. 
Step 2: $\quad$ By 84 , we have

$$
\begin{aligned}
\left|\mathbf{u}_{h}^{*}\right|_{1, \Omega_{h}}^{2}=\int_{\Omega_{h}} p_{h}^{*} \nabla \cdot \mathbf{u}_{h}^{*} & =-\left(\int_{\Omega_{h}} \frac{p_{h}^{*} q_{h}}{\left\|q_{h}\right\|_{\mathbb{Q}}}+\int_{\Omega_{h}} p_{h}^{*} \nabla \cdot \hat{\mathbf{u}}_{h}\right) \\
& \leqslant\left\|p_{h}^{*}\right\|_{\mathbb{Q}}+\left\|p_{h}^{*}\right\|_{\mathbb{Q}}\left\|\nabla \cdot \hat{\mathbf{u}}_{h}\right\|_{0, \Omega_{h}} \leqslant 2\left\|p_{h}^{*}\right\|_{\mathbb{Q}},
\end{aligned}
$$

using (83) and the fact that $\left\|\nabla \cdot \hat{\mathbf{u}}_{h}\right\|_{0, \Omega_{h}} \leqslant\left\|\hat{\mathbf{u}}_{h}\right\| \|_{\mathbb{V}}=1$. We also have, by using the inf-sup condition for the discrete Stokes problem [10], the following bound:

$$
\tilde{\beta}\left\|p_{h}^{*}\right\|_{\mathbb{Q}} \leqslant \sup _{\mathbf{v}_{h} \in \mathbb{V}_{h, 0}} \frac{\int_{\Omega_{h}} p_{h}^{*} \nabla \cdot \mathbf{v}_{h}}{\left\|\mathbf{v}_{h}\right\|_{1, \Omega_{h}}}=\sup _{\mathbf{v}_{h} \in \mathbb{V}_{h, 0}} \frac{\int_{\Omega_{h}} \nabla \mathbf{u}_{h}^{*}: \nabla \mathbf{v}_{h}}{\left\|\mathbf{v}_{h}\right\|_{1, \Omega_{h}}}=\left\|\mathbf{u}_{h}^{*}\right\|_{1, \Omega_{h}} .
$$

Hence, $\left\|p_{h}^{*}\right\|_{\mathbb{Q}} \leqslant(1 / \tilde{\beta})\left\|\mathbf{u}_{h}^{*}\right\|_{1, \Omega_{h}}$. Combining with $(85)$, we have

$$
\left\|\mathbf{u}_{h}^{*}\right\|\left\|_{\mathbb{V}} \leqslant c_{1}\right\| \mathbf{u}_{h}^{*} \|_{1, \Omega_{h}} \leqslant 2 c_{1} / \tilde{\beta}=: c_{2},
$$

because $\mathbf{u}_{h}^{*}$ has zero boundary data.

Step 3: Finally, let $\mathbf{u}_{h}:=\hat{\mathbf{u}}_{h}+\mathbf{u}_{h}^{*}$. By the previous steps, we know that $\left\|\mathbf{u}_{h}\left|\|\mathbb{V} \leqslant\| \hat{\mathbf{u}}_{h}\right|\right\| \mathbb{V}+$ $\left\|\mathbf{u}_{h}^{*}\right\| \|_{\mathbb{V}} \leqslant 1+c_{2}$, and using [84, we get the following inequality:

$$
\begin{aligned}
b\left(\mathbf{u}_{h}, q_{h}\right)+c\left(\mathbf{u}_{h}, \mu_{h}\right) & =-\int_{\Omega_{h}} q_{h}\left(\nabla \cdot \hat{\mathbf{u}}_{h}+\nabla \cdot \mathbf{u}_{h}^{*}\right)+\int_{\Gamma_{h}} \mu_{h}\left(\hat{\mathbf{u}}_{h} \cdot \boldsymbol{\nu}\right) \\
& \geqslant \int_{\Omega_{h}} \frac{q_{h}^{2}}{\left\|q_{h}\right\|_{\mathbb{Q}}}+\beta_{2}\left\|\mu_{h}\right\|_{\mathbb{M}} \geqslant c_{3}\left(\left\|q_{h}\right\|_{\mathbb{Q}}^{2}+\left\|\mu_{h}\right\|_{\mathbb{M}}^{2}\right)^{1 / 2} .
\end{aligned}
$$

The assertion follows with $\beta:=c_{3} /\left(1+c_{2}\right)$.

\subsection{Error estimates}

We introduce some notation used throughout this section:

$$
\begin{array}{rlrl}
\bar{p}=\frac{1}{|\Omega|} \int_{\Omega} p, & p^{*}=p-\bar{p}, & \bar{p}_{h}=\frac{1}{|\Omega|} \int_{\Omega} p_{h}, \quad p_{h}^{*}=p_{h}-\bar{p}_{h}, \\
\lambda^{*}=\lambda-\bar{p}, & \lambda_{h}^{*}=\lambda_{h}-\bar{p}_{h} .
\end{array}
$$

These new variables account for the aforementioned lack of uniqueness for both $p$ and $\lambda$. We prove the following error bound.

THEOREM 5.6 (Error estimates) Let $\Omega$ be of class $W_{\infty}^{2}, h_{0}$ be the limiting mesh size constant to tackle the variational crime described in Subsection 5.4, and $h_{1}$ be the limiting mesh size to ensure the inf-sup condition provided in Lemma 5.4. Then we have the following error estimates for all $h \leqslant \min \left(h_{0}, h_{1}\right)$ :

$$
\begin{aligned}
\left\|p^{*}-p_{h}^{*}\right\|_{\mathbb{Q}}^{2}+\left\|\lambda^{*}-\lambda_{h}^{*}\right\|_{\mathbb{M}}^{2} \preceq & \inf _{q_{h}^{*} \in \mathbb{Q}_{h, 0}}\left\|q_{h}^{*}-p^{*}\right\|_{\mathbb{Q}}^{2}+\inf _{\mu_{h} \in \Lambda_{h}}\left\|\mu_{h}-\lambda\right\|_{\mathbb{M}}^{2}+\left\|\mathbf{u}-\mathbf{u}_{h}\right\|_{\mathbb{V}}^{2}, \\
\left\|\mathbf{u}-\mathbf{u}_{h}\right\|_{\mathbb{V}}^{2} \preceq & \inf _{\mathbf{v}_{h} \in \mathbb{V}_{h}}\left\|\mathbf{u}-\mathbf{v}_{h}\right\|_{\mathbb{V}}^{2}+\inf _{q_{h}^{*} \in \mathbb{Q}_{h, 0}}\left\|q_{h}^{*}-p^{*}\right\|_{\mathbb{Q}}^{2} \\
& +\inf _{\mu_{h} \in \Lambda_{h}}\left(\left\langle\lambda-\mu_{h}, \mathbf{u} \cdot \boldsymbol{\nu}\right\rangle+\left\|\mu_{h}-\lambda\right\|_{\mathbb{M}}^{2}\right) .
\end{aligned}
$$

Proof. This is a direct consequence of Lemmas 5.11 and 5.12 below. 
The critical term $\left\langle\lambda-\mu_{h}, \mathbf{u} \cdot \boldsymbol{\nu}\right\rangle$ in estimate (90) restricts the rate of convergence of finite element solutions. In fact, we have the following corollary.

COROLlaRY 5.7 (Decay rate for continuous piecewise linear $\lambda$ ) Let $\left\{T_{\Omega_{h}}\right\}_{h>0}$ be a sequence of shape regular meshes. Assume that the solution $(\mathbf{u}, p, \lambda)$ of Problem 3.4 satisfies

$$
\mathbf{u} \in H^{2}(\Omega) \cap H^{2}(\Gamma), \quad p \in H^{1}(\Omega), \quad \lambda \in B V(\Gamma)
$$

Then, for all $h \leqslant \min \left(h_{0}, h_{1}\right)$, we have the estimate

$$
\begin{aligned}
\|\| \mathbf{u}-\mathbf{u}_{h}\left\|_{\mathbb{V}}+\right\| p^{*} & -p_{h}^{*}\left\|_{\mathbb{Q}}+\right\| \lambda^{*}-\lambda_{h}^{*} \|_{\mathbb{M}} \\
& \preceq h\left(\|\mathbf{u}\|_{H^{2}(\Omega) \cap H^{2}(\Gamma)}+\|p\|_{H^{1}(\Omega)}+\|\lambda\|_{B V(\Gamma)}\right)+\sqrt{\delta t} h\|\mathbf{u}\|_{H^{2}(\Gamma)},
\end{aligned}
$$

where the constant in ' $\preceq$ ' is independent of $h$ and $\delta t$.

Proof. We choose $\mathbf{v}_{h}=\pi_{\mathbb{V}_{h}} \mathbf{u}$ in (90) and $q_{h}^{*}=\pi_{\mathbb{Q}_{h}} p^{*}, \mu_{h}=\pi_{\Lambda_{h}} \lambda$ in (89)-90). Since $\pi_{\mathbb{Q}_{h}}$ is linear and preserves constants, the function $q_{h}^{*}$ has zero mean value. All the terms in (89)-(90) can be estimated via interpolation theory (recall Section 5.3), except for $\left\langle\lambda-\pi_{\Lambda_{h}} \lambda, \mathbf{u} \cdot \boldsymbol{\nu}\right\rangle$. Hence, we deduce

$$
\begin{aligned}
\left\|\mathbf{u}-\pi_{h} \mathbf{u}\right\|_{\mathbb{V}} & +\left\|p^{*}-\pi_{h} p^{*}\right\|_{\mathbb{Q}}+\left\|\lambda-\pi_{\Lambda_{h}} \lambda\right\|_{\mathbb{M}} \\
& \preceq h\left(\|\mathbf{u}\|_{H^{2}(\Omega)}+\|p\|_{H^{1}(\Omega)}+\|\lambda\|_{B V(\Gamma)}\right)+\sqrt{\delta t} h\|\mathbf{u}\|_{H^{2}(\Gamma)},
\end{aligned}
$$

because the $\mathbb{M}$ norm estimate follows from

$$
\left|\left\langle\lambda-\pi_{\Lambda_{h}} \lambda, \varphi\right\rangle\right| \leqslant\left\|\lambda-\pi_{\Lambda_{h}} \lambda\right\|_{L^{1}(\Gamma)}\|\varphi\|_{L^{\infty}(\Gamma)} \leqslant\left\|\lambda-\pi_{\Lambda_{h}} \lambda\right\|_{L^{1}(\Gamma)}\|\varphi\|_{H^{1}(\Gamma)}, \quad \forall \varphi \in H^{1}(\Gamma),
$$

and Sobolev embedding in 1-D; thus $\left\|\lambda-\pi_{\Lambda_{h}} \lambda\right\|_{\mathbb{M}} \preceq h\|\lambda\|_{B V(\Gamma)}$.

It remains to estimate $\left\langle\lambda-\pi_{\Lambda_{h}} \lambda, \mathbf{u} \cdot \boldsymbol{\nu}\right\rangle$, for which we only need to consider sides $S$ contained in $\Gamma_{h}$ where $\mathbf{u} \cdot \boldsymbol{\nu}$ changes sign, for the following reason. Let $S \in \mathrm{S}_{\Gamma}$ be such that $\mathbf{u} \cdot \boldsymbol{\nu}$ is not zero and has a definite sign over $S$. Then $\lambda=P_{\text {pin }} \operatorname{sgn}(\mathbf{u} \cdot \boldsymbol{\nu})$ is a fixed constant over $S$, so $\pi_{\Lambda_{h}} \lambda=\lambda$ over $S$ (i.e. $\left\langle\lambda-\pi_{\Lambda_{h}} \lambda, \mathbf{u} \cdot \boldsymbol{\nu}\right\rangle=0$ over $S$ ). Thus, let $S \in \mathrm{S}_{\Gamma}$ be so that $\mathbf{y} \in S$ and $(\mathbf{u} \cdot \boldsymbol{\nu})(\mathbf{y})=0$. Then

$$
\left|\int_{S}\left(\lambda-\pi_{\Lambda_{h}} \lambda\right) \mathbf{u} \cdot \boldsymbol{\nu}\right| \leqslant\left\|\lambda-\pi_{\Lambda_{h}} \lambda\right\|_{L^{1}(S)}\|\mathbf{u} \cdot \boldsymbol{\nu}\|_{L^{\infty}(S)} \preceq h^{2}\|\lambda\|_{B V(S)}\|\mathbf{u}\|_{W_{\infty}^{1}(S)},
$$

because $(\mathbf{u} \cdot \boldsymbol{\nu})(\mathbf{y})=0$. Adding over all such $S \in \mathrm{S}_{\Gamma}$ where $\mathbf{u} \cdot \boldsymbol{\nu}$ changes sign and applying Cauchy-Schwarz, we obtain

$$
\left|\left\langle\lambda-\pi_{\Lambda_{h}} \lambda, \mathbf{u} \cdot \boldsymbol{\nu}\right\rangle\right| \preceq h^{2}\|\lambda\|_{B V(\Gamma)}^{2}+h^{2}\|\mathbf{u}\|_{W_{\infty}^{1}(\Gamma)}^{2} .
$$

Since $\|\mathbf{u}\|_{W_{\infty}^{1}(\Gamma)} \preceq\|\mathbf{u}\|_{H^{2}(\Gamma)}$ the estimate 92 follows from 89 -90.

REMARK 5.8 (Regularity) The regularity assumed in (91) for $\mathbf{u}$ on $\Gamma$ is less restrictive than in 62) by Sobolev embedding. The condition $\lambda \in B V(\Gamma)$ is guaranteed by a stronger condition in the spirit of [11, Section 5]: $\lambda$ has bounded variation in the contact set and the number of points over which $\mathbf{u} \cdot \boldsymbol{\nu}$ changes from zero to nonzero is finite. 
REMARK 5.9 (Optimality) We demonstrate experimentally in Section 7 that the linear rate of convergence of 92 is not suboptimal for the polynomial degree used. This is due to the critical term $\left\langle\lambda-\pi_{\Lambda_{h}} \lambda, \mathbf{u} \cdot \boldsymbol{\nu}\right\rangle$, which plays a dominant role.

REMARK 5.10 (Decay rate for piecewise constant $\lambda$ ) In the proof of Corollary 5.7 we have not used that $\pi_{\Lambda_{h}} \lambda$ is piecewise linear, but rather that it is of first order in $L^{1}(\Gamma)$. Therefore, the same error estimates (92) are valid for piecewise constant $\pi_{\Lambda_{h}} \lambda$, provided (91) holds. In this case, $\pi_{\Lambda_{h}}$ is the standard $L^{2}(\Gamma)$ projection and (76) follows along the same lines as in the proof of Lemma 5.4. Interestingly, the computational results of Section 7 indicate that 92 is suboptimal as far as $\mathbf{u}$ and $p$ is concerned. This issue requires further study.

We now state Lemma 5.11 and 5.12 . Observing that

$$
b(\mathbf{v}, q)+c(\mathbf{v}, \mu)=b(\mathbf{v}, q+C)+c(\mathbf{v}, \mu+C)
$$

for all constants $C$, Lemma 5.11 is a special case of Lemma 2.7 in [51]; we omit the proof. Subsequently, we prove Lemma 5.12 which is a modification of Lemma 2.9 in [51].

Lemma 5.11 (Velocity error representation) Let $(\mathbf{u}, p, \lambda)$ solve the time-discrete Problem 3.4 and $\left(\mathbf{u}_{h}, p_{h}, \lambda_{h}\right)$ solve the fully discrete Problem 5.3. Then, for all $\left(\mathbf{v}_{h}, q_{h}, \mu_{h}\right) \in \mathbb{V}_{h} \times \mathbb{Q}_{h} \times \Lambda_{h}$,

$$
\begin{aligned}
a\left(\mathbf{v}_{h}-\mathbf{u}_{h}, \mathbf{v}_{h}-\mathbf{u}_{h}\right) \leqslant & B_{1}\left(q_{h}, \mu_{h}\right)+a\left(\mathbf{v}_{h}-\mathbf{u}, \mathbf{v}_{h}-\mathbf{u}_{h}\right)+b\left(\mathbf{v}_{h}-\mathbf{u}, p_{h}^{*}-p^{*}\right) \\
& +c\left(\mathbf{v}_{h}-\mathbf{u}, \lambda_{h}^{*}-\lambda^{*}\right)+b\left(\mathbf{u}_{h}-\mathbf{u}, p^{*}-q_{h}^{*}\right)+c\left(\mathbf{u}_{h}-\mathbf{u}, \lambda^{*}-\left(\mu_{h}-\bar{q}_{h}\right)\right),
\end{aligned}
$$

where

$$
B_{1}\left(q_{h}, \mu_{h}\right)=b\left(\mathbf{u}, p^{*}-q_{h}^{*}\right)+c\left(\mathbf{u}, \lambda^{*}-\left(\mu_{h}-\bar{q}_{h}\right)\right) .
$$

LEMMA 5.12 (Pressure-multiplier error representation) Let $\Omega$ be of class $W_{\infty}^{2}$ and let $h_{1}$ ensure the inf-sup condition as in Lemma 5.4 . Then

$$
\begin{gathered}
\left\|p^{*}-p_{h}^{*}\right\|_{\mathbb{Q}}+\left\|\lambda^{*}-\lambda_{h}^{*}\right\|_{\mathbb{M}} \leqslant \alpha_{1}\left(\left\|q_{h}^{*}-p^{*}\right\|_{\mathbb{Q}}+\left\|\mu_{h}-\lambda\right\|_{\mathbb{M}}+\left\|\mathbf{u}-\mathbf{u}_{h}\right\| \|_{\mathbb{V}},\right. \\
\left\|\mathbf{u}-\mathbf{u}_{h}\right\|_{\mathbb{V}}^{2} \leqslant \alpha_{2}\left(B_{1}\left(q_{h}, \mu_{h}\right)+\left\|q_{h}^{*}-p^{*}\right\|_{\mathbb{Q}}^{2}+\left\|\mu_{h}-\lambda\right\|_{\mathbb{M}}^{2}+\left\|\mathbf{u}-\mathbf{v}_{h}\right\|_{\mathbb{V}}^{2}\right),
\end{gathered}
$$

for all $\mathbf{v}_{h} \in \mathbb{V}_{h}, q_{h}^{*} \in \mathbb{Q}_{h, 0}, \mu_{h} \in \Lambda_{h}$, and $h \leqslant h_{1}$. Note that $\alpha_{1}$ and $\alpha_{2}$ depend on $\delta t$.

Proof. Using (93), along with (40) and (72), we get

$$
\begin{aligned}
b\left(\mathbf{v}_{h}, q_{h}^{*}-p_{h}^{*}\right)+c\left(\mathbf{v}_{h}, \eta_{h}-\lambda_{h}^{*}\right) & =b\left(\mathbf{v}_{h}, q_{h}^{*}\right)+c\left(\mathbf{v}_{h}, \eta_{h}\right)-b\left(\mathbf{v}_{h}, p_{h}\right)-c\left(\mathbf{v}_{h}, \lambda_{h}\right) \\
& =b\left(\mathbf{v}_{h}, q_{h}^{*}-p^{*}\right)+c\left(\mathbf{v}_{h}, \eta_{h}-\lambda^{*}\right)+a\left(\mathbf{u}_{h}-\mathbf{u}, \mathbf{v}_{h}\right)
\end{aligned}
$$

for all $q_{h} \in \mathbb{Q}_{h}, \eta_{h} \in \mathbb{M}_{h}$. So, combining the discrete inf-sup condition provided by Lemma 5.4 with 960) gives

$$
\left\|q_{h}^{*}-p_{h}^{*}\right\|_{\mathbb{Q}}+\left\|\eta_{h}-\lambda_{h}^{*}\right\|_{\mathbb{M}} \leqslant \frac{C_{1}}{\beta_{*}}\left(\left\|\mathbf{u}_{h}-\mathbf{u}\right\|_{\mathbb{V}}+\left\|q_{h}^{*}-p^{*}\right\|_{\mathbb{Q}}+\left\|\eta_{h}-\lambda^{*}\right\|_{\mathbb{M}}\right) .
$$

Starting with the left hand side of 94 and using a triangle inequality coupled with the choice $\eta_{h}:=\mu_{h}-\bar{p}$, and the definition of $\lambda^{*}:=\lambda-\bar{p}$, gives the rest of (94). 
As for (95), we start with Lemma 5.11 Using the coercivity of $a(\cdot, \cdot)$ (recall 399), CauchySchwarz, and the triangle inequality, we have

$$
\begin{aligned}
\left\|\mathbf{v}_{h}-\mathbf{u}_{h}\right\|_{\mathbb{V}}^{2} \leq & B_{1}\left(q_{h}, \mu_{h}\right)+\left\|\mathbf{v}_{h}-\mathbf{u}\right\|_{\mathbb{V}}^{2}+\left\|\mathbf{v}_{h}-\mathbf{u}\right\| \mathbb{\mathbb { V }}\left\|\mathbf{u}-\mathbf{u}_{h}\right\| \mathbb{V}_{\mathbb{V}} \\
& +\left\|\mathbf{v}_{h}-\mathbf{u}\right\| \mathbb{\mathbb { V }}\left\{\left\|p_{h}^{*}-p^{*}\right\|_{\mathbb{Q}}+\left\|\lambda_{h}^{*}-\lambda^{*}\right\|_{\mathbb{M}}\right\} \\
& +\left\|\mathbf{u}_{h}-\mathbf{u}\right\| \mathbb{\mathbb { V }}\left\{\left\|p^{*}-q_{h}^{*}\right\|_{\mathbb{Q}}+\left\|\lambda^{*}-\left(\mu_{h}-\bar{q}_{h}\right)\right\|_{\mathbb{M}}\right\}
\end{aligned}
$$

Employing $\left\|\mathbf{u}-\mathbf{u}_{h}\right\|_{\mathbb{V}}^{2} \leqslant 2\left|\left\|\mathbf{u}-\mathbf{v}_{h}\right\|_{\mathbb{V}}^{2}+2\right|\left\|\mathbf{v}_{h}-\mathbf{u}_{h}\right\|_{\mathbb{V}}^{2}$ and Young's inequality we get, by combining with (98) and (94), the following inequality:

$$
\begin{aligned}
\left\|\mathbf{u}-\mathbf{u}_{h}\right\|_{\mathbb{V}}^{2} \leq & \left\|\mathbf{u}-\mathbf{v}_{h}\right\|_{\mathbb{V}}^{2}+B_{1}\left(q_{h}, \mu_{h}\right) \\
& +\left\|q_{h}^{*}-p^{*}\right\|_{\mathbb{Q}}^{2}+\left\|\mu_{h}-\lambda\right\|_{\mathbb{M}}^{2}+\left\|p^{*}-q_{h}^{*}\right\|_{\mathbb{Q}}^{2}+\left\|\lambda^{*}-\left(\mu_{h}-\bar{q}_{h}\right)\right\|_{\mathbb{M}}^{2} .
\end{aligned}
$$

Finally, setting $\bar{q}_{h}:=\bar{p}$ gives us the velocity error estimate 95 .

\section{Solving the mixed system with inequality constraint}

We describe our iterative method for computing the solution of the nonlinear time-discrete Problem 3.4. Since our finite element spaces $\left(\mathbb{V}_{h}, \mathbb{Q}_{h}, \mathbb{M}_{h}\right)$ are conforming, the same analysis applies to the fully discrete problem [5.3]. For convenience, we recall that we are seeking $(\mathbf{u}, p, \lambda) \in \mathbb{V} \times \mathbb{Q} \times \Lambda$ such that

$$
\begin{array}{rlrl}
a(\mathbf{u}, \mathbf{v})-\int_{\Omega} p \nabla \cdot \mathbf{v}+\int_{\Gamma} \lambda(\mathbf{v} \cdot \boldsymbol{\nu})=\chi(\mathbf{v}), & & \forall \mathbf{v} \in \mathbb{V}, \\
\int_{\Omega} q \nabla \cdot \mathbf{u}=0, & \forall q \in \mathbb{Q}, \\
\int_{\Gamma}(\mu-\lambda) \mathbf{u} \cdot \boldsymbol{\nu} \leqslant 0, & \forall \mu \in \Lambda .
\end{array}
$$

We proceed to derive an Uzawa method, which takes advantage of an $L^{2}(\Gamma)$ projection property. First, observe that the variational inequality in 999 can be written as follows:

$$
0 \geqslant \int_{\Gamma}(\lambda-\omega)(\lambda-\mu), \quad \omega:=\lambda+\rho \mathbf{u} \cdot \boldsymbol{\nu} \quad(\rho>0)
$$

This implies that $\lambda$ is the $L^{2}$ projection of $\omega$ onto $\Lambda$, i.e. $\lambda=P_{\Lambda}(\lambda+\rho \mathbf{u} \cdot \boldsymbol{\nu})$. This suggests the following iterative method for solving the system 99 : given $\lambda_{0}=0$ and $j \geqslant 0$, let $\left(\mathbf{u}_{j}, p_{j}\right) \in \mathbb{V} \times \mathbb{Q}$ solve

$$
\begin{array}{rlrl}
a\left(\mathbf{u}_{j}, \mathbf{v}\right)-\int_{\Omega} p_{j} \nabla \cdot \mathbf{v}+\int_{\Gamma} \lambda_{j}(\mathbf{v} \cdot \boldsymbol{\nu}) & =\chi(\mathbf{v}), & \forall \mathbf{v} \in \mathbb{V}, \\
\int_{\Omega} q \nabla \cdot \mathbf{u}_{j}=0, & \forall q \in \mathbb{Q},
\end{array}
$$

and update the multiplier according to

$$
\lambda_{j+1}=P_{\Lambda}\left(\lambda_{j}+\rho \mathbf{u}_{j} \cdot \boldsymbol{\nu}\right) .
$$


LEMMA 6.1 (Convergence of the Uzawa method) Let $(\mathbf{u}, p, \lambda) \in \mathbb{V} \times \mathbb{Q} \times \Lambda$ be the solution of 99). Let $\left(\mathbf{u}_{j}, p_{j}, \lambda_{j}\right) \in \mathbb{V} \times \mathbb{Q} \times \Lambda$ be the solution of (100)-(101) for $j \geqslant 0$. Let $c_{a}$ be the coercivity constant in 39. Assume that the relaxation parameter $\rho$ satisfies

$$
\rho<2 D_{\text {visc }} \quad \text { if } D_{\text {visc }}>0, \quad \text { or } \rho<2 c_{a} \delta t \quad \text { otherwise. }
$$

Then the sequence $\left(\mathbf{u}_{j}, p_{j}, \lambda_{j}\right)$ converges in $\mathbb{V} \times \mathbb{Q} \times \Lambda$ to $(\mathbf{u}, p, \lambda)$, in the sense that

$$
\mathbf{u}_{j} \rightarrow \mathbf{u}, \quad p_{j}^{*} \rightarrow p^{*}, \quad \lambda_{j}^{*} \rightarrow \lambda^{*},
$$

where $p^{*}, \lambda^{*}$ are defined in (88) and $p_{j}^{*}, \lambda_{j}^{*}$ are defined similarly, namely $p_{j}^{*}:=p_{j}-\bar{p}_{j}, \lambda_{j}^{*}:=$ $\lambda_{j}-\bar{p}_{j}$. Moreover, if $\lambda=P_{\text {pin }}$ on a set of positive measure, and $\lambda=-P_{\text {pin }}$ on a set of positive measure, then we also have $p_{j} \rightarrow p$ and $\lambda_{j} \rightarrow \lambda$, i.e. full convergence.

Proof. The analysis follows Glowinski [30], but is adapted to our mixed formulation. Let

$$
\delta \lambda_{j}=\lambda_{j}-\lambda, \quad \delta p_{j}=p_{j}-p, \quad \delta \mathbf{u}_{j}=\mathbf{u}_{j}-\mathbf{u}
$$

be the errors between the iterates and the exact solution. Because the $L^{2}(\Gamma)$ projection, $P_{\Lambda}$, is a contraction (see [27]), the update of the multiplier $\lambda_{j+1}$ in (101) implies that $\left\|\delta \lambda_{j+1}\right\|_{0, \Gamma} \leqslant$ $\left\|\delta \lambda_{j}+\rho \delta \mathbf{u}_{j} \cdot \nu\right\|_{0, \Gamma}$. Squaring both sides and moving terms around gives

$$
\left\|\delta \lambda_{j}\right\|_{0, \Gamma}^{2}-\left\|\delta \lambda_{j+1}\right\|_{0, \Gamma}^{2} \geqslant \rho\left(-2 \int_{\Gamma} \delta \lambda_{j}\left(\delta \mathbf{u}_{j} \cdot \boldsymbol{\nu}\right)-\rho\left\|\delta \mathbf{u}_{j} \cdot \boldsymbol{\nu}\right\|_{0, \Gamma}^{2}\right) .
$$

Now we need to take advantage of the variational formulation. The linearity of the first two equations in 99) as well (100) gives, for all $\mathbf{v} \in \mathbb{V}$ and $q \in \mathbb{Q}$,

$$
\begin{aligned}
&\left(\frac{\alpha}{\delta t}+\beta\right)\left(\int_{\Omega} \delta \mathbf{u}_{j} \cdot \mathbf{v}+\right.\left.\gamma \int_{\Omega}\left(\nabla \cdot \delta \mathbf{u}_{j}\right)(\nabla \cdot \mathbf{v})\right)+D_{\mathrm{visc}} \int_{\Gamma}\left(\delta \mathbf{u}_{j} \cdot \boldsymbol{\nu}\right)(\mathbf{v} \cdot \boldsymbol{\nu}) \\
&+\delta t \int_{\Gamma} \nabla_{\Gamma} \delta \mathbf{u}_{j} \cdot \nabla_{\Gamma} \mathbf{v}-\int_{\Omega} \delta p_{j} \nabla \cdot \mathbf{v}+\int_{\Gamma} \delta \lambda_{j} \mathbf{v} \cdot \boldsymbol{\nu}=0, \\
& \int_{\Omega} q \nabla \cdot \delta \mathbf{u}_{j}=0 .
\end{aligned}
$$

Setting the test functions $\mathbf{v}=\delta \mathbf{u}_{j}$ and $q=\delta p_{j}$ in (103), and using the coercivity (39) of $a(\cdot, \cdot)$, we find

$$
-\int_{\Gamma} \delta \lambda_{j}\left(\delta \mathbf{u}_{j} \cdot \boldsymbol{\nu}\right) \geqslant c_{a}\left(r_{0}\left\|\delta \mathbf{u}_{j}\right\|_{H(\operatorname{div}, \Omega)}^{2}+\delta t\left\|\delta \mathbf{u}_{j}\right\|_{1, \Gamma}^{2}\right)+D_{\mathrm{visc}}\left\|\delta \mathbf{u}_{j} \cdot \boldsymbol{\nu}\right\|_{0, \Gamma}^{2},
$$

where $c_{a}, r_{0}>0$ are uniform constants. Combining (104) with (102) gives

$$
\begin{aligned}
\left\|\delta \lambda_{j}\right\|_{0, \Gamma}^{2}-\left\|\delta \lambda_{j+1}\right\|_{0, \Gamma}^{2} & \\
& \geqslant \rho\left\{2 c_{a}\left(r_{0}\left\|\delta \mathbf{u}_{j}\right\|_{H(\operatorname{div}, \Omega)}^{2}+\delta t\left\|\delta \mathbf{u}_{j}\right\|_{1, \Gamma}^{2}\right)+\left(2 D_{\mathrm{visc}}-\rho\right)\left\|\delta \mathbf{u}_{j} \cdot \boldsymbol{\nu}\right\|_{0, \Gamma}^{2}\right\},
\end{aligned}
$$

Hence, if $D_{\text {visc }}>0$ and $\rho<2 D_{\text {visc }}$, then $\left\{\left\|\delta \lambda_{j}\right\|_{0, \Gamma}^{2}\right\}_{j=0}^{\infty}$ is a decreasing sequence of nonnegative numbers, so it converges. If instead $D_{\text {visc }}=0$, using $\left\|\delta \mathbf{u}_{j} \cdot \boldsymbol{\nu}\right\|_{0, \Gamma} \leqslant\left\|\delta \mathbf{u}_{j}\right\|_{1, \Gamma}$ we obtain

$$
\left\|\delta \lambda_{j}\right\|_{0, \Gamma}^{2}-\left\|\delta \lambda_{j+1}\right\|_{0, \Gamma}^{2} \geqslant \rho\left(2 c_{a} \delta t-\rho\right)\left\|\delta \mathbf{u}_{j}\right\|_{1, \Gamma}^{2} .
$$


Ergo, $\rho<2 c_{a} \delta t$ in order to guarantee the convergence of $\left\{\left\|\delta \lambda_{j}\right\|_{0, \Gamma}^{2}\right\}_{j=0}^{\infty}$. Both cases mean the right hand side of (105) goes to zero as $j$ goes to $\infty$. Therefore, as $j \rightarrow \infty$,

$$
\mathbf{u}^{j} \rightarrow \mathbf{u} \quad \text { in } \mathbb{V} .
$$

In order to show the convergence of $\delta \lambda_{j}$ and $\delta p_{j}$, we use the inf-sup condition. Using [103, and the definition of the bilinear forms 36 , 377, gives

$$
b\left(\mathbf{v}, \delta p_{j}\right)+c\left(\mathbf{v}, \delta \lambda_{j}\right)=-a\left(\delta \mathbf{u}_{j}, \mathbf{v}\right), \quad \forall \mathbf{v} \in \mathbb{V} .
$$

By the constant shift property 993 , and continuity of $a(\cdot, \cdot)$, we can write

$$
\frac{b\left(\mathbf{v}, p_{j}^{*}-p^{*}\right)+c\left(\mathbf{v}, \lambda_{j}^{*}-\lambda^{*}\right)}{\|\mathbf{v}\| \mathbb{V}} \leqslant a_{0}\left\|\delta \mathbf{u}_{j}\right\| \mathbb{V}, \quad \forall \mathbf{v} \in \mathbb{V} .
$$

It then follows by the continuous version of the inf-sup (76) (recall Remark 4.7) that there is a constant $c_{0}>0$ such that

$$
\left\|\mathbf{u}_{j}-\mathbf{u}\right\|_{\mathbb{V}} \geqslant c_{0}\left(\left\|p_{j}^{*}-p^{*}\right\|_{\mathbb{Q}}^{2}+\left\|\lambda_{j}^{*}-\lambda^{*}\right\|_{\mathbb{M}}^{2}\right)^{1 / 2} .
$$

So, by [107], we have $p_{j}^{*} \rightarrow p^{*}$ and $\lambda_{j}^{*} \rightarrow \lambda^{*}$ in their respective norms.

Clearly, if $\lambda$ does not touch both the positive and negative constraints of $\Lambda$ (i.e. $\pm P_{\text {pin }}$ ), it is possible to add a small constant to both $p_{j}$ and $\lambda_{j}$ and still have the above convergence. This is expected.

For the case when $\lambda$ does contact both the positive and negative constraints on sets of positive measure, we now show that $\bar{p}_{j} \rightarrow \bar{p}$, i.e. full convergence of $p_{j}$ and $\lambda_{j}$. Here, we use the fact that $\lambda \in L^{\infty}(\Gamma)$. Given $\epsilon>0$, there exists $N$ such that for all $j \geqslant N,\left\|\lambda_{j}^{*}-\lambda^{*}\right\|_{\mathbb{M}}<\epsilon$. By the definition of the $\mathbb{M}$ norm 26, we have

$$
-\epsilon<\int_{\Gamma}\left(\lambda_{j}^{*}-\lambda^{*}\right) \eta=\int_{\Gamma}\left(\lambda_{j}-\lambda-\alpha_{j}\right) \eta<\epsilon, \quad \forall \eta \in \mathbb{G}:\|\eta\|_{\mathbb{G}}=1,
$$

with $\alpha_{j}:=\bar{p}_{j}-\bar{p}$. Assume that $\lambda=P_{\text {pin }}$ on an open set $\Gamma_{+} \subset \Gamma$. Let $\eta \geqslant 0$ be a smooth function in $\mathbb{G}$ that vanishes outside of $\Gamma_{+}$such that $\|\eta\|_{\mathbb{G}}=1$. Let $C_{+}=\int_{\Gamma} \eta$; note that $C_{+}>0$ is independent of $\epsilon$ because $\eta$ only depends on $\lambda$. By [110], we have

$$
\alpha^{j} C_{+}<P_{\text {pin }} \int_{\Gamma_{+}} \eta-P_{\text {pin }} \int_{\Gamma_{+}} \eta+\epsilon=\epsilon,
$$

where we used the fact that $\lambda_{j} \leqslant P_{\text {pin }}$; ergo, $\alpha_{j}<\epsilon / C_{+}$. By a similar argument, if $\lambda=-P_{\text {pin }}$ on an open set $\Gamma_{-} \subset \Gamma$, then we get $\alpha_{j}>-\epsilon / C_{-}$with $C_{-}>0$. Hence,

$$
\left|\alpha^{j}\right|<\frac{\epsilon}{\min \left(C_{+}, C_{-}\right)}, \quad \forall j \geqslant N(\epsilon) .
$$

Letting $\epsilon \rightarrow 0$ implies that $\bar{p}_{j}-\bar{p}=\alpha_{j} \rightarrow 0$ as $j \rightarrow \infty$. Putting everything together, we conclude that $p_{j} \rightarrow p$ and $\lambda_{j} \rightarrow \lambda$, as asserted.

REMARK 6.2 (Nonuniqueness in practice) In our numerical implementation, we leave the constant $\alpha_{j}$ free. This may change the mean value of $p$ and $\lambda$ when the droplet is completely pinned but does not affect the velocity and evolution of the droplet.

\section{Numerical rates of convergence}

We show some experimental rates of convergence for a particular exact solution of Problem 3.4 


\subsection{Exact solution}

The error analysis we give in Section 5.6 relates to the error between the semi-discrete and fully discrete equations for a single time step. Therefore, we present an 'exact' solution for the semidiscrete problem (4)-(7).

7.1.1 Strong form PDE. All constants in the PDE are set to unity. In addition, we take liberty in modifying the right-hand-side data of the problem for convenience in creating an exact solution. In short, the PDE we will solve is defined as

$$
\begin{aligned}
\mathbf{u}+\nabla p-\mathbf{F}=0 & \text { in } \Omega, \\
\nabla \cdot \mathbf{u}=0 & \text { in } \Omega, \\
\lambda \in \operatorname{sgn}(\mathbf{u} \cdot \boldsymbol{\nu}) & \text { on } \Gamma, \\
p-E-\lambda+\left(\Delta_{\Gamma} \mathbf{u}\right) \cdot \boldsymbol{\nu}=0 & \text { on } \Gamma, \\
g-\left(\Delta_{\Gamma} \mathbf{u}\right) \cdot \boldsymbol{\tau}=0 & \text { on } \Gamma,
\end{aligned}
$$

where the domain $\Omega$ is depicted in Figure 3 . Note that we keep the critical term $\Delta_{\Gamma} \mathbf{u}$ in the strong form PDE. The functions $\mathbf{F}, E$, and $g$ are given data for the problem (yet to be determined). Items to note in 112 are:

- The explicit curvature of the domain appearing in (11) has been removed. If we had kept that term, it would merely have changed the particular $E$ that we actually use.

- We include an extra tangential term in the last equation of (112). Recall the extra condition stated in (13), which says that $\Delta_{\Gamma} \mathbf{u} \cdot \boldsymbol{\tau}=0$. For convenience in defining the exact solution, we change this condition to have nonzero forcing (i.e. $g \neq 0$ ). Again, we are only taking liberty with the right-hand-side data.

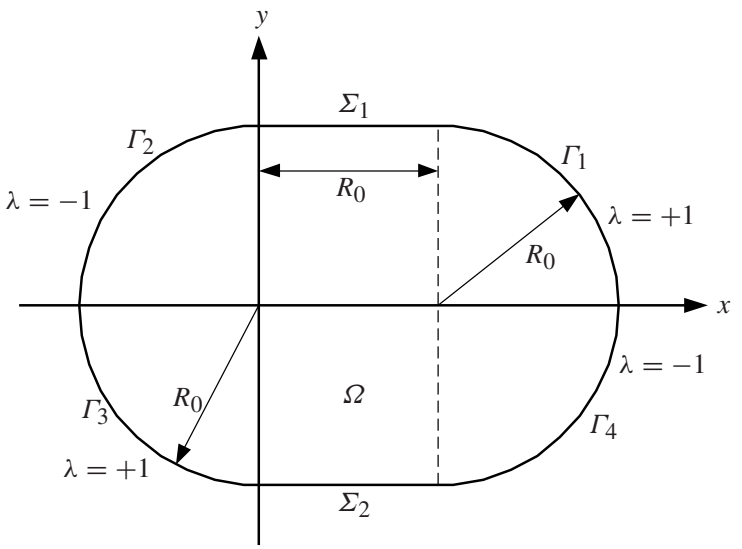

FIG. 3. 2-D droplet $\Omega$ with boundary $\Gamma=\partial \Omega=\bigcup_{i=1}^{4} \Gamma_{i} \cup \bigcup_{j=1}^{2} \Sigma_{j}$ and $R_{0}=1 / 2$. An exact solution $(\mathbf{u}, p, \lambda)$ is defined on $\Omega$.

7.1.2 Exact velocity, pressure, and pinning. The exact velocity $\mathbf{u}=\left(u_{1}, u_{2}\right)$ is

$$
u_{1}=\sin \left(\frac{\pi}{2} \frac{y}{R_{0}}\right), \quad u_{2}=0, \quad \text { in } \Omega .
$$


The exact pressure is

$$
p=-x \sin \left(\frac{\pi}{2} \frac{y}{R_{0}}\right) \quad \text { in } \Omega
$$

The exact $\lambda$ is

$$
\lambda= \begin{cases}1 & \text { on } \Gamma_{1} \cup \Gamma_{3}, \\ -1 & \text { on } \Gamma_{2} \cup \Gamma_{4}, \\ -\frac{y}{R_{0}} \cos \left(\pi \frac{x}{R_{0}}\right) & \text { on } \Sigma_{1} \cup \Sigma_{2} .\end{cases}
$$

The exact velocity $\mathbf{u}$ and pressure $p$ are nontrivial smooth functions. The pinning variable $\lambda$ jumps at the extreme ends of the domain $\Omega$ (i.e. $\lambda$ is discontinuous at $\left(-R_{0}, 0\right),\left(2 R_{0}, 0\right)$, and is smooth everywhere else).

\subsubsection{Exact given data. The exact body force is}

$$
\mathbf{F}=\left(0,-\left(\frac{\pi}{2} \frac{x}{R_{0}}\right) \cos \left(\frac{\pi}{2} \frac{y}{R_{0}}\right)\right) \quad \text { in } \Omega .
$$

The exact $g$ is defined from the tangential condition in 112 :

$$
g=\left(\Delta_{\Gamma} \mathbf{u}\right) \cdot \boldsymbol{\tau} \quad \text { on } \Gamma .
$$

The exact $E$ is defined from the normal condition in (112):

$$
E=p-\lambda+\left(\Delta_{\Gamma} \mathbf{u}\right) \cdot \boldsymbol{\nu} \quad \text { on } \Gamma .
$$

The body force $\mathbf{F}$ is smooth, whereas $g$ is piecewise smooth and continuous. The normal forcing $E$ is piecewise smooth and discontinuous at the same two points as $\lambda$.

\subsection{Error plots}

This section presents plots of the error decay between the exact and approximate solutions of the system (112). We first discuss the case when $\lambda \in \mathcal{P}_{1}$ (piecewise linear) for two different meshes in Section 7.2.1 One mesh has a node that conforms to the points of discontinuity in $\lambda$ (see Section 7.1.2), and is thus fitted, whereas the other mesh is non-fitted to the discontinuity of $\lambda$. In Section 7.2.2. we show the error decay when $\lambda \in \mathcal{P}_{0}$ (piecewise constant).

7.2.1 Continuous piecewise linear $\lambda$. In Figure 4, we show experimental orders of convergence of the fully discrete solution $\left(\mathbf{u}_{h}, p_{h}, \lambda_{h}\right)$ to the exact solution $(\mathbf{u}, p, \lambda)$ of $(112)$ when $\lambda \in \mathcal{P}_{1}$. The error estimates in Corollary 5.7 appear to be optimal because the decay of pressure is linear for non-fitted meshes. Nonetheless, the pressure error is $O\left(h^{2}\right)$ for fitted meshes. The decay rate of velocity $O\left(h^{4 / 3}\right)$ is a bit better than predicted regardless of whether meshes are fitted or not.

7.2.2 Discontinuous piecewise constant $\lambda$. In Figure 5, we show experimental orders of convergence when $\lambda \in \mathcal{P}_{0}$. The error estimates behave much better than expected and we do not have a complete explanation for this. This may be linked to the exact solution that we constructed. See the caption of Figure 5 for more details. 

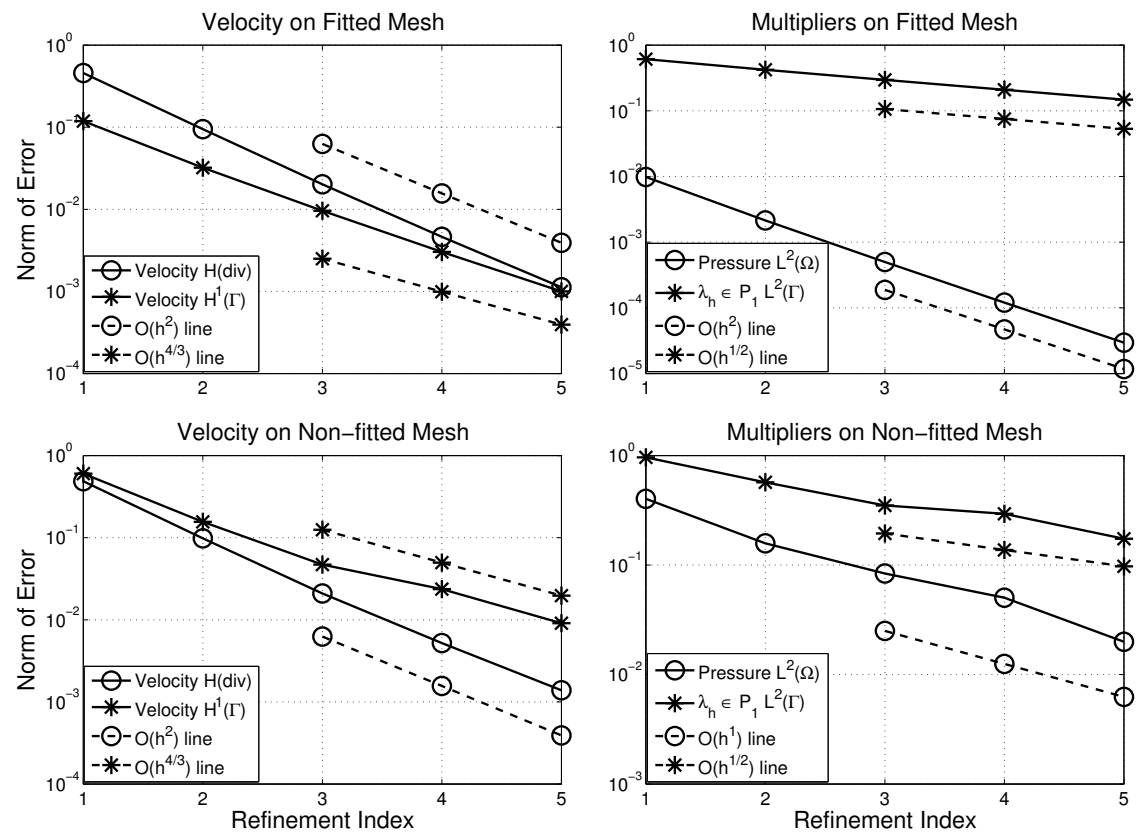

FIG. 4. Error plots for $\lambda_{h} \in \mathcal{P}_{1}$. The top row corresponds to using a mesh with nodes lying at both points of discontinuity in $\lambda$. The bottom row is for the non-fitted mesh case (i.e. the discontinuity occurs inside an edge element for each mesh in the refinement sequence). The left column plots the error in velocity; the right column gives the error for both pressure and pinning. For convenience of computation, we measure the error $\lambda-\lambda_{h}$ in $L^{2}(\Gamma)$ instead of in $\mathbb{M}$. Because $\lambda_{h}$ is continuous and $\lambda$ is discontinuous, the order of decay is only $O\left(h^{1 / 2}\right)$ for both meshes by standard interpolation theory. Note the error estimate in Corollary 5.7 is for the $\mathbb{M}$ norm. When the mesh is fitted, the pressure has better convergence $O\left(h^{2}\right)$. For the non-fitted mesh (a more generic case), pressure is $O(h)$ (optimal). The convergence of velocity in $H^{1}(\Gamma)$ appears to be slightly better (approximately $O\left(h^{4 / 3}\right)$ ).

\section{Conclusion}

We have presented a mixed finite element method for a model of 2-D droplet motion driven by electrowetting [56, 55, 57]. Our method uses a stable semi-implicit discretization of curvature and incorporates local contact line pinning forces via a variational inequality as well as viscous damping. These two loss mechanisms are responsible for the remarkable space-time agreement between our simulations and actual experiments, which are reported in [57]. We have shown existence and uniqueness results for the semi-discrete and fully discrete problems as well as an error analysis for the finite element approximation. We have proven the convergence of an Uzawa method for solving the time-discrete system at a single time step, and concluded with some experimental convergence results that explore the limits and optimality of the theory.

Extending our results to the fully continuous case is not trivial. For instance, the techniques developed in [13] are seemingly not applicable in this context. Indeed, the lack of regularity of the $\operatorname{sgn}(\cdot)$ function in (2) prevents us from deriving energy estimates in more regular spaces as proposed in [13]. 

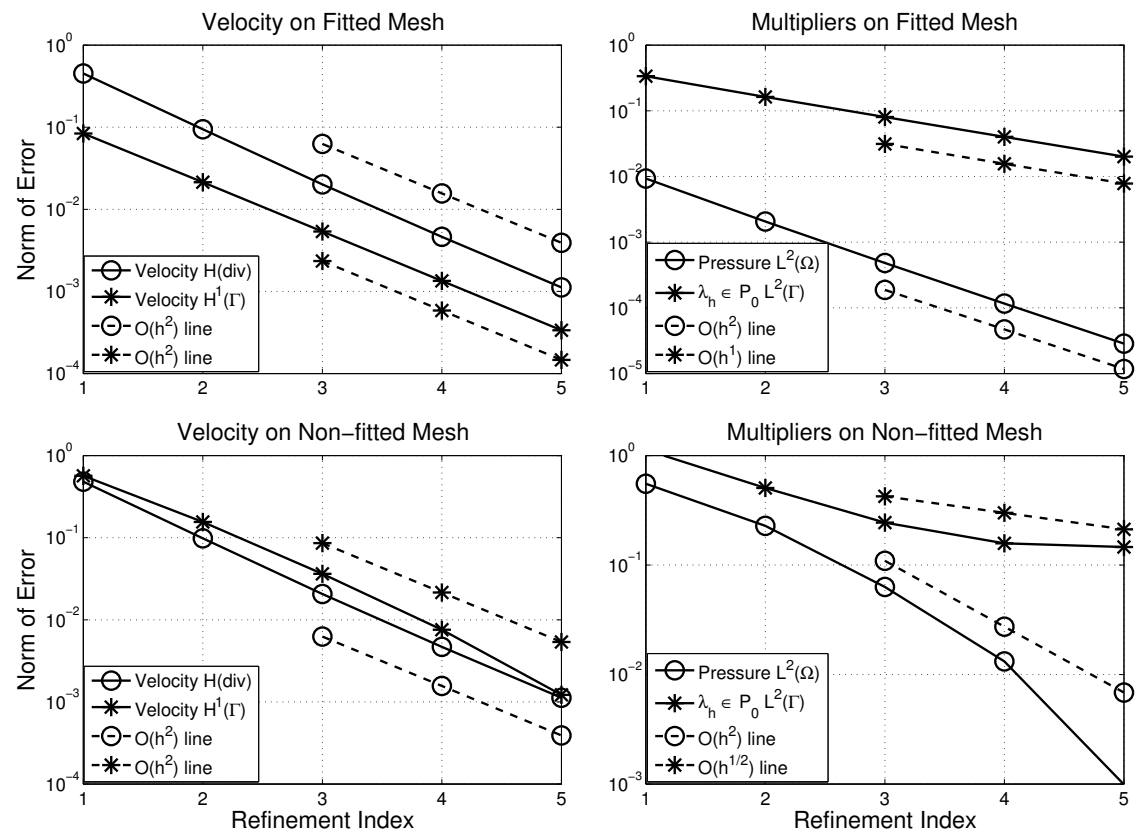

FIG. 5. Error plots for $\lambda_{h} \in \mathcal{P}_{0}$ with the same format as in Figure 4 The convergence is $O\left(h^{2}\right)$ for both velocity and pressure for both fitted and non-fitted meshes. In the case of a fitted mesh (top row), $\lambda_{h}$ is able to exactly capture the discontinuity of the exact $\lambda$, which may play a role in the improved convergence of the other variables. However for the non-fitted mesh, the convergence of $\lambda_{h}$ is only $O\left(h^{1 / 2}\right)$, which does not seem to affect the convergence of velocity and pressure. We cannot fully explain this improved convergence. Also note the behavior of pressure in the bottom right plot.

\section{Acknowledgments}

We wish to acknowledge funding support. Walker was partially supported by the NSF-VIGRE grant of the Department of Mathematics, University of Maryland, College Park, in addition to support from NSF-RTG grant DMS-0602235 and DOE grant DE-FG02-88ER25053. Bonito was partially supported by NSF grants DMS-0914977, DMS-0505454, and DMS-0807811. Nochetto was partially supported by NSF grants DMS-0505454 and DMS-0807811, with current support by NSF grant CBET 0754983 (program managers Maria Burka and William Schultz).

\section{REFERENCES}

1. Adams, R. A., \& Fournier, J. J. F. Sobolev Spaces. 2nd ed., Pure Appl. Math. 140, Elsevier (2003). Zbl 1098.46001 MR 2424078

2. Arnold, D. N., Brezzi, F., \& Fortin, M. A stable finite element for the Stokes equations. Calcolo 21 (1984), 337-344. Zbl 0593.76039 MR 0799997

3. B ̈̈NSCH, E. Finite element discretization of the Navier-Stokes equations with a free capillary surface. Numer. Math.. 88 (2001), 203-235. Zbl 0985.35060 MR 1826211 
4. Batchelor, G. K. An Introduction to Fluid Dynamics. Cambridge Univ. Press, New York (1967). Zbl 0152.44402 MR 1744638

5. Bedekar, A. S., Jenkins, J. W., \& Sundaram, S. A computational model for the design of electrowetting on dielectric (ewod) systems. In: NSTI Nanotechnology Conference \& Trade Show (Anaheim, CA, 2005), Session Presentation.

6. Berge, B. Électrocapillarité et mouillage de films isolants par l'eau. C. R. Acad. Sci. Paris Sér. II 317 (1993), 157-163.

7. Bonito, A., Nochetto, R. H., \& Pauletti, M. S. Geometrically consistent mesh modification. In preparation.

8. Braess, D. Finite Elements: Theory, Fast Solvers, and Applications in Solid Mechanics. 2nd ed., Cambridge Univ. Press (2001). Zbl 0976.65099 MR 1827293

9. Brenner, S. C., \& Scott, L. R. The Mathematical Theory of Finite Element Methods. 2nd ed., Springer, New York (2002). Zbl 1012.65115 MR 1894376

10. BrezzI, F., \& Fortin, M. Mixed and Hybrid Finite Element Methods. Springer, New York (1991). Zbl 0788.73002 MR 1115205

11. Brezzi, F., Hager, W. W., \& Raviart, P. A. Error estimates for the finite element solution of variational inequalities: Part II. Mixed methods. Numer. Math. 31 (1978), 1-16. Z Zbl 0427.65077 MR 0508584

12. Burdon, R. S. Surface Tension and the Spreading of Liquids. Cambridge Univ. Press, Cambridge (1949). Zbl 0028.04102

13. Cheng, C. H. A., Coutand, D., \& ShKoller, S. Navier-Stokes equations interacting with a nonlinear elastic biofluid shell. SIAM J. Math. Anal. 39 (2007), 742-800. Zbl 1138.74022 MR 2349865

14. CHO, S. K., \& KIM, C.-J. Particle separation and concentration control for digital microfluidic systems. In: The 16th Annual IEEE International Conference on MEMS (Kyoto, 2003), 686-689.

15. Chо, S. K., Moon, H., \& KIM, C.-J. Creating, transporting, cutting, and merging liquid droplets by electrowetting-based actuation for digital microfluidic circuits. J. Microelectromech. Systems 12 (2003), 70-80.

16. Clément, P. Approximation by finite element functions using local regularization. R.A.I.R.O. Anal. Numér. 9 (1975), 77-84. Zbl 0368.65008 MR 0400739

17. Darhuber, A. A., Davis, J. M., Troian, S. M., \& Reisner, W. W. Thermocapillary actuation of liquid flow on chemically patterned surfaces. Phys. Fluids 15 (2003), 10150-10153.

18. De Gennes, P.-G., Brochard-Wyart, F., \& QuÉré, D. Capillarity and Wetting Phenomena: Drops, Bubbles, Pearls, Waves. Springer, New York (2004). Zbl 1139.76004

19. Do Carmo, M. P. Differential Geometry of Curves and Surfaces. Prentice Hall, Upper Saddle River, NJ (1976). Zbl 0326.53001 MR 0394451

20. Drelich, E. J., Laskowski, J. S., \& Mittal, K. L. (eds.) Apparent and Microscopic Contact Angles. VSP, Zeist (2000).

21. Dussan, E. B. On the ability of drops or bubbles to stick to non-horizontal surfaces of solids. Part 2. Small drops or bubbles having contact angles of arbitrary size. J. Fluid Mech. 151 (1985), 1-20. Zbl 0574.76102

22. Duvaut, G., \& Lions, J.-L. Inequalities in Mechanics and Physics. Springer, New York (1976). Zbl 0331.35002 MR 0521262

23. DzIUK, G. An algorithm for evolutionary surfaces. Numer. Math. 58 (1990), 603-611. Z Zbl 0714.65092 MR 1083523

24. Eck, C., Fontelos, M., Grün, G., Klingbeil, F., \& Vantzos, O. On a phase-field model for electrowetting. Interfaces Free Bound. 11 (2009), 259-290. Zbl 1167.35553 MR 2511642

25. Evans, L. C. Partial Differential Equations. Amer. Math. Soc., Providence, RI (1998). Zbl 0902.35002 MR 1625845 
26. Fortner, N., Shapiro, B., \& Hightower, A. Modeling and passive control of channel filling for micro-fluidic networks with thousands of channels. In: American Institute of Aeronautics and Astronautics (AIAA) 33rd AIAA Fluid Dynamics Conference and Exhibit (Orlando, FL, 2003).

27. Friedman, A. Variational Principles and Free-Boundary Problems. Wiley (1982). Zbl 0564.49002 MR 0679313

28. GALDI, G. P. An Introduction to the Mathematical Theory of the Navier-Stokes Equations. Vol. I. Springer Tracts in Natural Philosophy 38, Springer, New York (1994). Zbl 0949.35004 MR 1284205

29. Girault, V., \& Raviart, P. A. Finite Element Methods for Navier-Stokes Equations: Theory and Algorithms. Springer, Berlin (1986). Zbl 0585.65077 MR 0851383

30. Glowinski, R. Numerical Methods for Nonlinear Variational Problems. Springer, New York (1984). Zbl 0536.65054 MR 0737005

31. Gong, J., FAN, S. K., \& KIM, C. J. Portable digital microfluidics platform with active but disposable lab-on-chip. In: 17th IEEE International Conference on Micro Electro Mechanical Systems (MEMS) (Maastricht, 2004), IEEE Press, 355-358.

32. HE, B., \& LEE, J. Dynamic wettability switching by surface roughness effect. In: The 16th Annual IEEE International Conference on MEMS (Kyoto, 2003), 120-123.

33. Hele-Shaw, H. S. The flow of water. Nature (London) 58 (1898), 34-36.

34. Henning, A., Staudinger, U., Eichhom, K.-J., Sahre, K., Rogalli, M., Stamm, M., Neumann, A. W., \& GRUndKe, K. Contact angle hysteresis: study by dynamic cycling contact angle measurements and variable angle spectroscopic ellipsometry on polyimide. Langmuir 20 (2004), 66856691.

35. Johnny, J. F., \& Robbins, M. O. Motion of a contact line on a heterogeneous surface. J. Chem. Phys. 92 (1990), 3206-3212.

36. LAng, S. Real and Functional Analysis. 3rd ed., Grad. Texts in Math. 142, Springer (1993). Zbl 0831.46001 MR 1216137

37. LAX, P. D. Functional Analysis. Wiley-Interscience (2002). Zbl 1009.47001 MR 1892228

38. LENOIR, M. Optimal isoparametric finite elements and error estimates for domains involving curved boundaries. SIAM J. Numer. Anal. 23 (1986), 562-580. Zbl 0605.65071 MR 0842644

39. Lienemann, J., Greiner, A., \& Korvink, J. G. Modeling, simulation, and optimization of electrowetting. IEEE Trans. Computer-Aided Design of Integrated Circuits and Systems 25 (2006), 234247.

40. Lions, J.-L., \& Magenes, E. Non-Homogeneous Boundary Value Problems. Vol. 1, Springer (1972). Zbl 0223.35039 MR 0350177

41. Lions, J.-L., \& Stampacchia, G. Variational inequalities. Comm. Pure Appl. Math. 20 (1967), 493519. Zbl 0152.34601 MR 0216344

42. Lu, H.-W., Glasner, K., Bertozzi, A. L., \& KIM, C.-J. A diffuse interface model for electrowetting drops in a Hele-Shaw cell. J. Fluid Mech. 590 (2007), 411-435. Z Zbl 1141.76482

43. Minnema, L., Barneveld, H. A., \& Rinkel, P. D. An investigation into the mechanism of water treeing in polyethylene high voltage cables. IEEE Trans. Electrical Insulators 15 (1980), 461-472.

44. Mohseni, K., \& Dolatabadi, A. An electrowetting microvalve: Numerical simulation. Ann. New York Acad. Sci. 1077 (2006), 415-425.

45. Moon, H., Wheeler, A. R., Garrell, R. L., Loo, J. A., \& Kim, C.-J. On-chip sample preparation by electrowetting-on-dielectric digital microfluidics for matrix assisted laser desorption/ionization mass spectrometry. In: Proceedings of IEEE MEMS (Miami, FL, 2005), 859-862.

46. Robbins, M. O., \& JoAnny, J. F. Contact angle hysteresis on random surfaces. Europhys. Lett. 3 (1987), 729-735.

47. Satoh, W., Loughran, M., \& Suzuki, H. Microfluidic transport based on direct electrowetting. J. Appl. Phys. 96 (2004), 835-841. 
48. Schaffer, E., \& Wong, P. Z. Dynamics of contact line pinning in capillary rise and fall. Phys. Rev. Lett. 80 (1998), 3069-3072.

49. ScOTT, L. R., \& ZHANG, S. Finite element interpolation of nonsmooth functions satisfying boundary conditions. Math. Comput. 54 (1990), 483-493. Zbl 0696.65007 MR 1011446

50. Shapiro, B., Moon, H., Garrell, R., \& Kim, C.-J. Equilibrium behavior of sessile drops under surface tension, applied external fields, and material variations. J. Appl. Phys. 93 (2003), 5794-5811.

51. Slimane, L., Bendali, A., \& Laborde, P. Mixed formulations for a class of variational inequalities. Math. Modelling Numer. Anal. 38 (2004), 177-201. Zbl 1100.65059 MR 2073936

52. Temam, R. Navier-Stokes Equations. AMS Chelsea Publ., Providence, RI (2001). Zbl 0568.35002 MR 0769654

53. Verfürth, R. Finite element approximation of incompressible Navier-Stokes equations with slip boundary condition. Numer. Math. 50 (1987), 697-721. Zbl 0596.76031 MR 0884296

54. WALKer, S. W. Modeling, simulating, and controlling the fluid dynamics of electro-wetting on dielectric. PhD thesis, Univ. of Maryland, College Park (2007).

55. WAlKer, S. W., \& Shapiro, B. A control method for steering individual particles inside liquid droplets actuated by electrowetting. Lab on a Chip 5 (2005), 1404-1407.

56. WAlker, S. W., \& Shapiro, B. Modeling the fluid dynamics of electrowetting on dielectric (ewod). J. Microelectromech. Systems 15 (2006), 986-1000.

57. Walker, S. W., Shapiro, B., \& Nochetto, R. H. Electrowetting with contact line pinning: Computational modeling and comparisons with experiments. Phys. Fluids 21 (2009), no. 10, 102103.

58. Wheeler, A. R., Moon, H., Kim, C.-J., Loo, J. A., \& GArrell, R. L. Electrowetting-based microfluidics for analysis of peptides and proteins by matrix-assisted laser desorption/ionization mass spectrometry. Analytical Chemistry 76 (2004), 4833-4838.

59. Wolfram, E., \& FAUst, R. Wetting, Spreading and Adhesion. Chapter "Liquid Drops on a Tilted Plate, Contact Angle Hysteresis and the Young Contact Angle". Academic Press, Leicestershire (1978). 